\title{
Sociotechnical safeguards for genomic data privacy
}

Zhiyu Wan (1) 1,2,3,9, James W. Hazel 1,4,9, Ellen Wright Clayton (10 ${ }^{1,4,5}$, Yevgeniy Vorobeychik ${ }^{6}$, Murat Kantarcioglu ${ }^{7}$ and Bradley A. Malin 1,2,3,8凶

Abstract $\mid$ Recent developments in a variety of sectors, including health care, research and the direct-to-consumer industry, have led to a dramatic increase in the amount of genomic data that are collected, used and shared. This state of affairs raises new and challenging concerns for personal privacy, both legally and technically. This Review appraises existing and emerging threats to genomic data privacy and discusses how well current legal frameworks and technical safeguards mitigate these concerns. It concludes with a discussion of remaining and emerging challenges and illustrates possible solutions that can balance protecting privacy and realizing the benefits that result from the sharing of genetic information.

There are many stories in the media highlighting the multitude of ways by which genomic data are now relied upon, including in basic research, clinical care, discovering relatives and ancestral origins, tracking down criminals, and identification of victims. At the same time, numerous reports from around the world illustrate that some people are concerned about how genomic information that relates to them are used, often stated as challenges to privacy. These apprehensions do have some foundation as people can suffer harm if data about them are used in ways they do not agree with, for example, to examine ancestry ${ }^{1}$ or to create commercial products $^{2}$ without the individual's approval, or if the data are used in a manner that causes an individual to suffer adverse consequences such as stigmatization ${ }^{3}$, disruption of familial relationships ${ }^{4,5}$ or loss of employment or insurance. However, the law provides limited, patchy protection $^{6,7}$.

The concept of privacy and its protection has many facets ${ }^{8}$. People may wish to control how genomic data about them are used but, in many cases, they only have the choice to opt in (or opt out) based on the terms contained in a consent form or a service agreement ${ }^{9}$, which frequently goes unread ${ }^{10,11}$. In other instances, people may not have any choice at all about how genomic data about them are used, such as when data are deemed to be anonymised in accordance with the General Data Protection Regulation (GDPR) ${ }^{12}$ of the European Union (EU), de-identified in accordance with the Health Insurance Portability and Accountability Act of 1996 (HIPAA $)^{13-15}$ or considered non-human subject data in accordance with the Common Rule for the Protection of Human Research Participants ${ }^{16}$ in the United States. Another aspect of privacy is the right to solitude (often voiced as the right to be left alone), a principle first formalized in legal circles in the late $1800 \mathrm{~s}^{17}$, which could include the right not to be (re)contacted about ancillary findings generated from genomic testing or discovery-driven investigations into existing genomic data sets ${ }^{18,19}$ or by previously unknown relatives ${ }^{20,21}$.

Yet, the right to privacy has never been absolute, in part because many uses of these data, such as clinical care, research, exploring ancestry, finding relatives and identifying criminal suspects and victims of mass casualties, can be valued by users, other stakeholders, or society at large. For example, even though physicians have strong ethical and legal duties of confidentiality that require them not to disclose patients' information to others, these obligations are not unconditional because the law has created numerous exceptions such as for public health reporting ${ }^{22}$ or in criminal investigations.

Although the tension between privacy and data utility raises an array of ethical issues ${ }^{23-25}$ regarding when genomic data can be accessed and used, this Review focuses on the primary tools that are applied to define and protect these boundaries: law (as instantiated in statutes, regulatory regimes and case law), policy and technology. Several reviews on genomic data privacy have been published over the years in response to the evolution of approaches to intrude upon, and protect, privacy. Initially, Malin appraised the robustness of genetic data de-identification ${ }^{26}$. This study was followed by Erlich and Narayanan who analysed and categorized computational methods for re-identification, in light of new techniques for surname inference, and potential risk mitigation techniques ${ }^{27}$. Naveed et al. reviewed the privacy and security threats that arise over the course of the genomic data lifecycle, from data generation to its end uses ${ }^{28}$. Wang et al. studied the technical and ethical aspects of genetic privacy ${ }^{29}$. Arellano et al. reviewed 


\section{Blockchains}

A blockchain is a decentralized digital ledger of records, called blocks, that are linked together using cryptography and are distributed across a peer-to-peer network of computers. policies and technologies for protecting the privacy of biomedical data in general ${ }^{30}$. More recently, Mittos et al. systematically reviewed privacy-enhancing technologies for genomic data and particularly highlighted the challenges associated with using cryptography to maintain privacy over a long period of time ${ }^{31}$. Grishin et al. reviewed the emerging cryptographic tools for protecting genomic privacy with a focus on blockchains ${ }^{32}$. Bonomi et al. reviewed privacy challenges as well as technical research opportunities for genomic data applications such as direct-to-consumer genetic testing (DTC-GT) and forensic investigations ${ }^{33}$. Similarly, numerous articles have addressed the incomplete and inconsistent protection that the law provides from harms to individuals and groups in different settings ${ }^{3,19,34-36}$.

Our Review diverges from prior work in that we consider it essential to discuss the legal and technological perspectives together. This is because technological interventions can heighten, but also ameliorate, legal risks, whereas some laws provide control or protect people from downstream harm from data use, thereby opening the door to different and perhaps less stringent technological protections. Moreover, recent disruptions associated with mandates for data sharing ${ }^{37,38}$, the DTC-GT revolution and the coronavirus disease 2019 (COVID-19) pandemic - events that have dramatically accelerated the collection and use of genomic data ${ }^{39-41}$ - have dramatically changed the social environment in which genomic data are obtained and used. Blending legal and technical protections in a holistic ecosystem of genomic data is challenging because protections are interconnected but vary in the environments in which they were developed, the stakeholders involved and their underlying assumptions. To demystify the connections among and the assumptions behind different legal and technical protections, we partition the ecosystem into four settings: health care, research, DTC and forensic settings.

In this Review, we begin with a brief overview of attacks on privacy in the context of genomic data sharing and subsequently discuss both how to mitigate privacy risks (through technical and legal safeguards) as well as the consequences of failing to do so effectively. Next, we categorize legal protections according to different settings since each setting tends to have unique laws and policies; meanwhile, we identify settings where each technical protection was first introduced and/or has been frequently applied. We consider the particular challenges that can arise in the research setting itself.

\footnotetext{
Author addresses

${ }^{1}$ Center for Genetic Privacy and Identity in Community Settings, Vanderbilt University

Medical Center, Nashville, TN, USA.

${ }^{2}$ Department of Computer Science, Vanderbilt University, Nashville, TN, USA.

${ }^{3}$ Department of Biomedical Informatics, Vanderbilt University Medical Center, Nashville, TN, USA.

${ }^{4}$ Center for Biomedical Ethics and Society, Vanderbilt University, Nashville, TN, USA.

${ }^{5}$ Vanderbilt University Law School, Nashville, TN, USA.

${ }^{6}$ Department of Computer Science and Engineering, Washington University in St. Louis, St. Louis, MO, USA.

${ }^{7}$ Department of Computer Science, University of Texas at Dallas, Richardson, TX, USA.

${ }^{8}$ Department of Biostatistics, Vanderbilt University Medical Center, Nashville, TN, USA.

${ }^{9}$ These authors contributed equally: Zhiyu Wan, James W. Hazel.
}

We then note that genomics researchers also need an appreciation of the larger ecology of the flows of genomic data outside the research and health-care settings in light of their impact on data privacy and public opinion and thus ultimately on public support for genomic research. Thus, we discuss DTC-GT, the obligations that companies that provide these tests owe to users, and the consequences of use by consumers to find relatives and by law enforcement to find criminal suspects. For reference, FIG. 1 illustrates an overview of privacy intrusions and safeguards in the ecology of genomic data flows, and TABLE 1 summarizes various aspects of the technical literature featured in this Review. In our discussions, a first party refers to the individual to whom the data correspond, whereas a second party refers to the organization (or individual) who collects and/or uses the data for a purpose that the first party is made aware of. By contrast, third parties refer to users (or recipients) of data who have the ability to communicate with the second party only and might include malicious attackers. Examples of third parties include researchers who access data from an existing research study or a pharmaceutical company that partners with a DTC-GT company. We conclude with a discussion of what legal revisions and technical advances may be warranted to balance privacy protection with the benefits to individuals, commercial entities, researchers and society that result from flows of genomic data.

\section{Privacy intrusions and protections Privacy intrusions}

Individuals may suffer harm when data about them are used without their permission in ways they do not agree with. In contrast to summary data aggregated across many participants, individual-level data that identify the people to whom they pertain, not surprisingly, pose a greater risk of harm to the person. For example, breaches of identified data might reveal a health condition that the participant had wished not to become public or cause them to suffer adverse consequences such as reputational damage or loss of employment, insurance, or other economic goods ${ }^{3}$. These disclosures can occur when data holders lose the data, for instance, by misplacing an unencrypted laptop, or when third parties deliberately attack large, identified data collections; therefore, security becomes particularly important when conducting research using identified data.

Much research using genomic data, however, is conducted with additional types of data, such as demographics, social and behavioural determinants of health, and phenotypic information at the molecular and/or clinical level (for example, data derived from electronic health records), from which standard identifying information have been removed. Yet, there has been a vigorous debate about whether genomic data can be de-identified or anonymised on its own or in combination with the accompanying individual information. Over the years, a number of investigators have famously demonstrated their ability to re-identify individuals whose data have been used without common identifiers for genomics research. The following provides a summary of these attacks. 


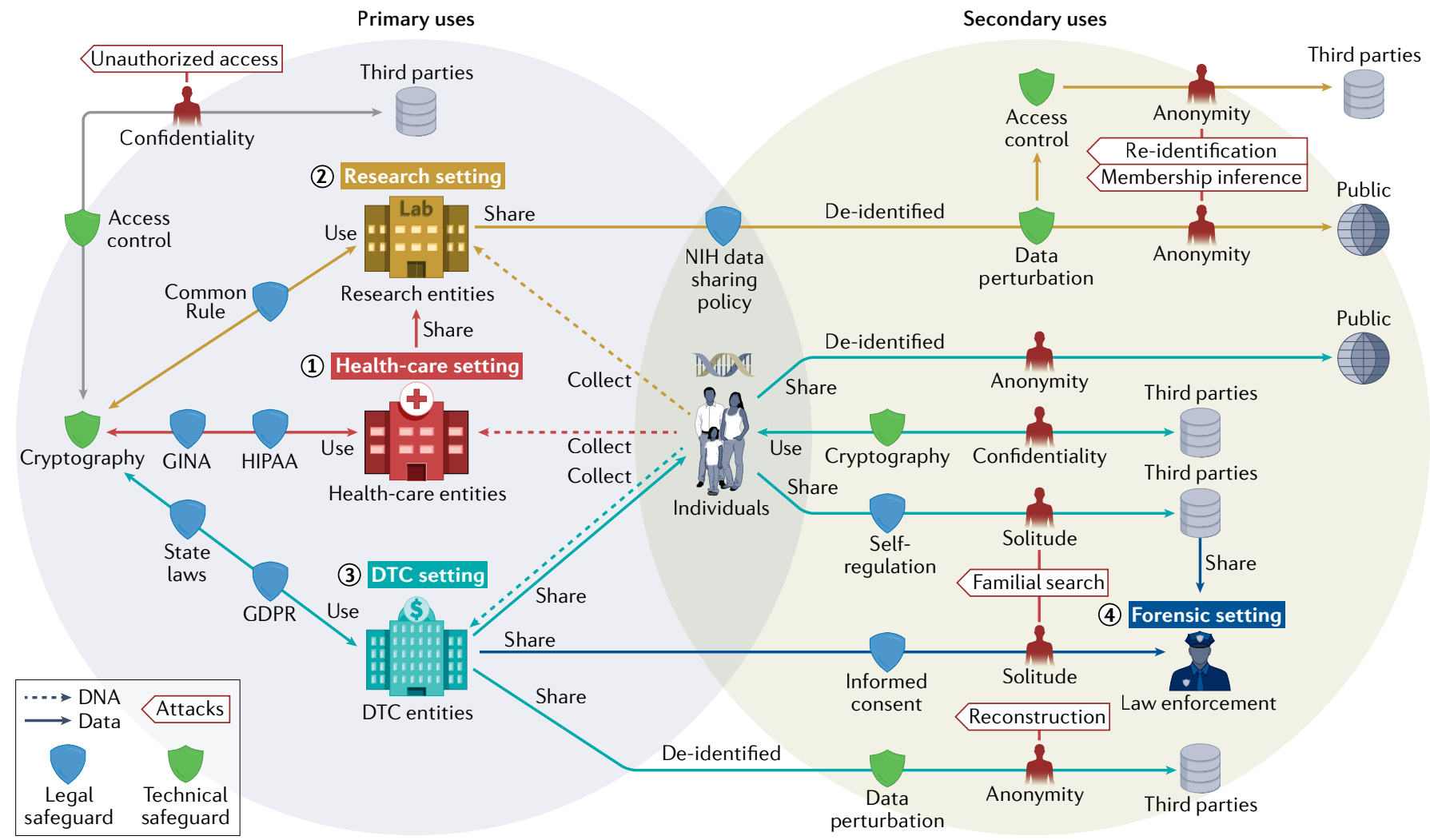

Fig. 1 | An overview of privacy intrusions and safeguards in genomic data flows. The four routes of genomic data flow (as indicated by the arrow colours) represent four settings in which data are used or shared: health care (red), research (gold), direct-to-consumer (DTC; green) and forensic (dark blue). The grey line represents a combination of the first three settings. In the health-care setting, data collected by a health-care entity (for example, Vanderbilt University Medical Center) are protected by the Genetic Information Nondiscrimination Act of 2008 (GINA) ${ }^{128}$ and the Health Insurance Portability and Accountability Act of 1996 (HIPAA) ${ }^{116,117}$ for primary uses. In the research setting, data collected by a research entity (for example, 1000 Genomes Project, Electronic Medical Records and Genomics (eMERGE) network or All of Us Research Program) are primarily protected by the Common Rule ${ }^{14,124}$ for primary uses and protected by the US National Institutes of Health $(\mathrm{NIH})$ data sharing policy ${ }^{37,38}$ for secondary uses. In the DTC setting, data collected by a DTC entity are protected by the European Union's General Data Protection Regulation (GDPR) ${ }^{12}$ and/or the US state privacy laws (for example, California Consumer Privacy Act ${ }^{130}$, California Privacy Rights Act ${ }^{131}$ or Virginia Consumer Data Protection $\mathrm{Act}^{132}$ ) for primary uses and protected

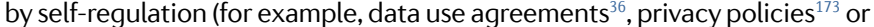
terms of service ${ }^{174}$ ) for secondary uses. In the forensic setting, data shared with law enforcement are protected by informed consent ${ }^{192}$. A first party refers to the individual to whom the data correspond, whereas a second party refers to the organization (or individual) who collects and/or uses the data for a purpose that the first party is made aware of. By contrast, third parties refer to users (or recipients) of data who have the ability to communicate with the second party only and might include malicious attackers. Examples of third parties include researchers who access data from an existing research study or a pharmaceutical company that partners with a DTC genetic testing company. The data flow from a DTC entity to a research entity is represented by the arrow at the bottom. Confidentiality is mostly concerned when data are being used, whereas anonymity and solitude are mostly concerned when data are being shared. Specifically, cryptographic tools ${ }^{31}$ protect confidentiality against unauthorized access attacks, whereas access control ${ }^{27}$ and data perturbation approaches ${ }^{83}$ protect anonymity against privacy intrusions such as re-identification and membership inference attacks. We simplify the figure by omitting the impacts of GDPR and data use agreements in the research setting.
Short tandem repeats (STRs). Short tandemly repeated DNA sequences that occur when two or more nucleotides (A, T, C or G) are repeated and the repeated sequences are adjacent to each other.
Re-identification. Sharing individual-level genomic data, even without explicit identifiers, creates an opportunity for re-identification ${ }^{42}$. For example, a data recipient could infer phenotypic information from genomic data that may be leveraged for re-identification purposes ${ }^{27,43}$. In one study, researchers re-identified individuals in a data set of whole-genome sequences by predicting visual traits, including eye and skin colour ${ }^{44}$. Similarly, genomic attributes might be inferred from phenotypic traits (for example, physically observable disorders ${ }^{45}$, visual traits $^{46,47}$ or 3D facial structures ${ }^{48}$ ) for re-identification purposes, although the actual power of these attacks is debatable $e^{47,49,50}$. In addition, known pedigree structures may be leveraged to re-identify genomic records ${ }^{51}$.

Moreover, potential identifiers may be inferred from the demographic information that is often shared with genomic data, through linkage to other readily accessible data sources. In 2013, participants of the Personal Genome Project ${ }^{52}$ were re-identified by Sweeney et al. by linking these participants' data records to publicly available voter registration lists using demographic attributes $^{53}$. In the same year, Gymrek et al. re-identified certain participants of the 1000 Genomes Project by first inferring surnames from short tandem repeats (STRs) on the Y chromosome, which, in combination with other demographics, were then linked to identified public resources ${ }^{54}$.

Membership inference. In genome-phenome investigations, such as genome-wide association studies (GWAS), researchers commonly publish only summary statistics that are useful for meta-analyses ${ }^{55}$. However, in 2008, 
Table 1 | A taxonomy of technical research articles on genomic data privacy featured in this Review

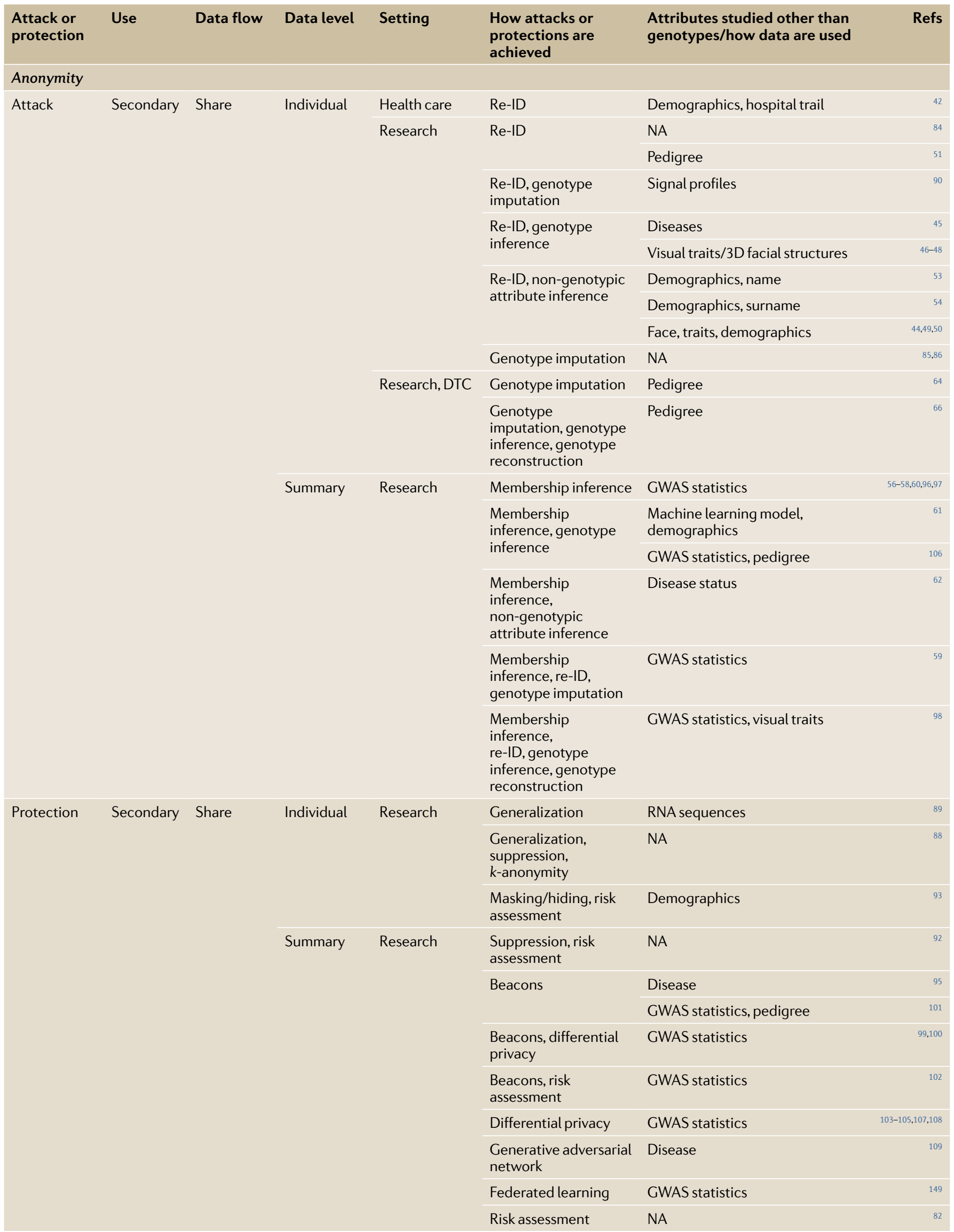


Table 1 (cont.) | A taxonomy of technical research articles on genomic data privacy featured in this Review

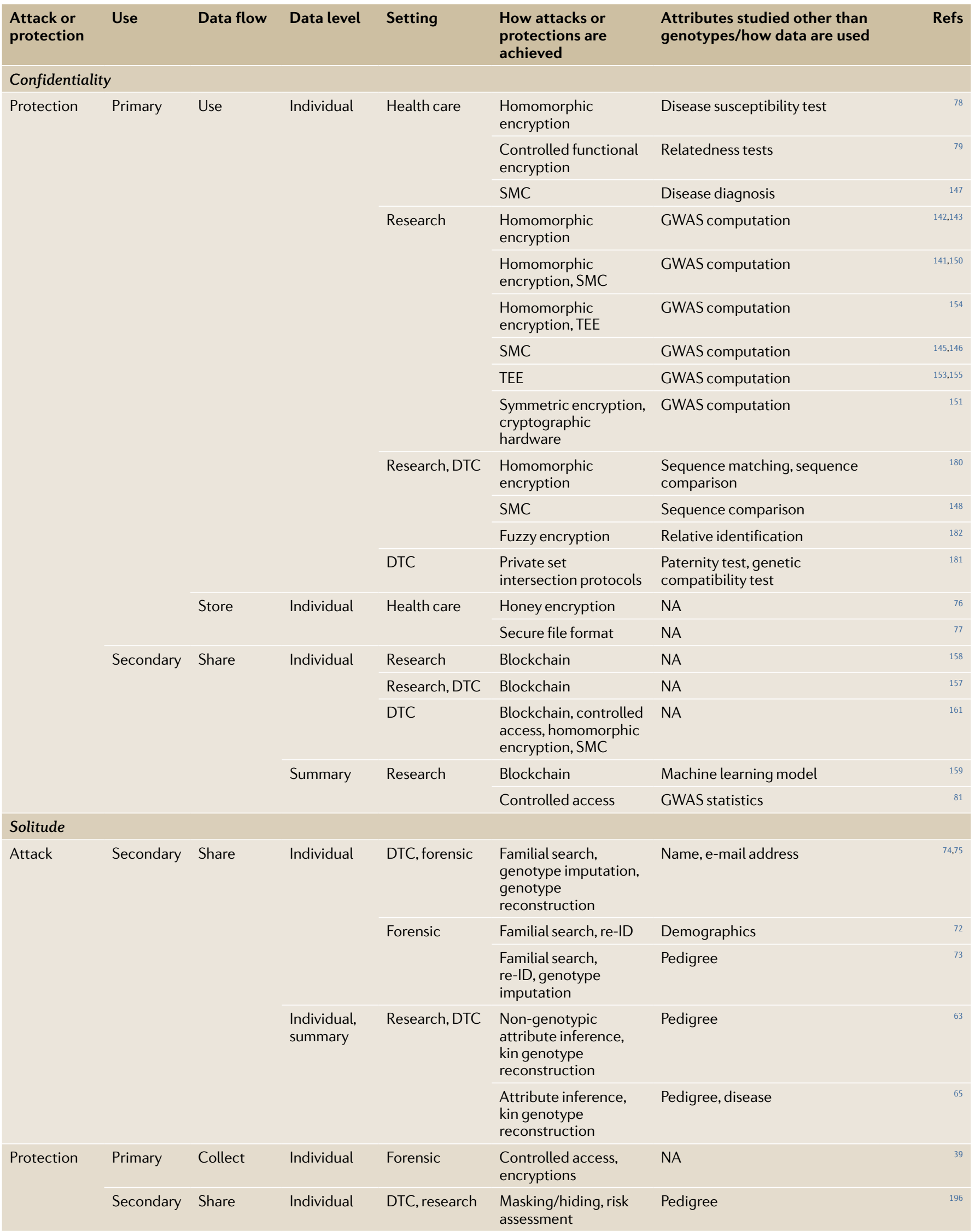

DTC, direct-to-consumer; GWAS, genome-wide association study; ID, identification; NA, not applicable; SMC, secure multiparty computation; TEE, trusted execution environment. 


\section{Phenome \\ The complete set of all phenotypes expressed by an organism as a result of genetic variation in populations. A phenotype is an individual's observable traits such as height, eye colour, blood type, skin colour, hair colour, specific personality characteristics or specific diseases.}

Genome-wide association studies

(GWAS). Observational studies in which genetics research scientists associate specific genetic variations with traits of interest, particularly human diseases. For human disease studies, this method scans the genomes from many different people and looks for genetic markers (for example, singlenucleotide polymorphisms) that occur more frequently in people with a particular disease than in people without the disease.

\section{Summary statistics} Numbers that give a quick and simple description of a set of records in a data set (for example, mean, median, minimum value, maximum value and standard deviation) A typical example of a summary statistic in a GWAS is a minor allele frequency.

Forensic or investigative genetic genealogy

(FGG/IGG). A process in which law enforcement seeks to exploit public databases or utilize the services of a directto-consumer genetic testing company for forensic purposes.

Single-nucleotide polymorphism

(SNP). The most common form of DNA variation that occurs when a single nucleotide (A, T, $\mathrm{C}$ or $\mathrm{G}$ ) at a specific position in the genome differs sufficiently (for example, $1 \%$ or more) in a species' population

Linkage disequilibrium Non-random correlations among neighbouring alleles. This occurs due to infrequent recombination events between nearby genomic loci, and hence the alleles are typically co-inherited by the next generation.

\section{Genotype imputation} A process of estimating missing genotypes from a haplotype or genotype reference panel.
Homer et al. demonstrated that GWAS summary statistics are vulnerable to membership inference attacks ${ }^{56}$, whereby it is possible to discover an identified target's participation in the GWAS as part of a potentially sensitive group. Although the power of this attack was questioned by other researchers ${ }^{57}$, subsequent studies showed that the inference power can be further improved by leveraging statistics based on allele frequencies ${ }^{58}$, correlation ${ }^{59}$ and regression coefficients ${ }^{60}$. Furthermore, parameters in machine learning (ML) models trained on individuallevel genomic data sets have the potential to disclose the genotypes and memberships of the participants ${ }^{61}$. Identifying an individual's membership in a GWAS data set could also reveal the participant's sensitive clinical information such as disease status ${ }^{62}$.

Reconstruction and familial search. Due to the similarity of relatives' genomic records, even if someone's genomic record has never been shared or even generated, their genotypes and predispositions to certain diseases ${ }^{63}$ can be inferred to a certain degree from their relatives' shared genotypes $^{64}$. Recently, more powerful reconstruction attacks have been proposed to infer individuals' genotypes and phenotypes from their relatives' genotypes and phenotypes ${ }^{65,66}$.

In April 2018, the US Federal Bureau of Investigation (FBI) used genomic data from a cold case to arrest a suspected serial murderer known as the Golden State Killer. In this case, law enforcement officers used crime-scene DNA from the then-unidentified suspect and uploaded the sequence data to GEDmatch, a publicly accessible genomic database. Through a process known as long-range familial search, whereby relatives can be identified based on shared blocks of DNA sequence, they found the suspect's third cousin. From this starting point in the suspect's wider family, law enforcement officers were then able to make further enquiries, reconstruct a family tree and subsequently trace the suspect. Although this case demonstrated the potential of the forensic use of familial search, now known as forensic or investigative genetic genealogy (FGG/IGG), it sparked privacy concerns ${ }^{67}$. Acknowledging these concerns, in May 2019, GEDmatch provided users with the opportunity to opt in to allow their data to be used for investigating violent crimes $^{68}$. By May 2020, most (81\%) of GEDmatch's 1.4 million users still have not opted in ${ }^{69}$, and users concerned about privacy did delete their data ${ }^{70}$. Potentially, users who uploaded data to GEDmatch (or a similar database) and their relatives may still be reached out using the long-range familial search technique by anyone (for example, law enforcement officers or hackers) who obtained their genomic data elsewhere ${ }^{71}$. A study that received a great deal of attention predicted that, in a database of 1 million individuals, $60 \%$ of searches using genome data from individuals of European descent as search queries will result in finding a third cousin or closer match to the targeted individuals due to the high number of individuals of European ancestry already in the database ${ }^{72}$. With the help of correlations between two types of genetic markers (that is, single-nucleotide polymorphism (SNP) and STR markers), the detection of relatives in genomic databases becomes even easier ${ }^{73}$. Although most records in these databases are not disclosed to end-users, stronger attacks have aimed to reconstruct records in a database by uploading strategically generated artificial records $\mathrm{s}^{74,75}$.

\section{Technical protections against intrusions}

Security controls. An important element of protecting privacy is preventing access to data by those who are not entitled to them. Some attacks targeting genomic data can be prevented by applying standard security controls (for example, access control ${ }^{27}$ and cryptographic tools ${ }^{31,76-79}$ ) and restricting access to selected trusted recipients ${ }^{27}$. For example, in response to attacks demonstrated by Gymrek et al. ${ }^{54}$ and Homer et al..$^{56}$, the US National Institutes of Health (NIH) and the Wellcome Trust moved certain demographics about the participants ${ }^{80}$ as well as GWAS summary statistics ${ }^{81}$ into access-controlled databases. Subsequent studies found the attack to be less powerful under more realistic assumptions ${ }^{82}$, which contributed to the NIH's decision in 2018 to derestrict public access to genomic summary statistics $^{33}$.

If de-identified data need to be shared with an untrusted third party or the public, the privacy of individuals to whom these data correspond can be protected by perturbing ${ }^{83}$ (that is, limiting or altering) the data. In the following sections and FIG. 2, we illustrate four approaches for technical protection that perturb data (that is, transformation, aggregation, obfuscation and synthetic data generation) with examples in the context of genomic data sharing.

Data transformation. Some have suggested that the number of released genetic variants should be limited because, among millions of SNPs in a person's genome, less than 100 statistically independent SNPs are required to identify each person uniquely ${ }^{84}$. However, protecting a genomic data set by hiding a set of genetic variants may not be very effective due to correlations among genetic variants (known as linkage disequilibrium) ${ }^{85}$ and well-established genotype imputation techniques ${ }^{86}$.

To thwart re-identification through linkage in general, Sweeney introduced $k$-anonymity ${ }^{87}$, a data transformation model, to ensure that each record in a released data set is equivalent to no fewer than $(k-1)$ other records with the same quasi-identifying values (that is, those which can be relied upon for linkage). Initially developed to address demographics, it was subsequently shown that this model could be applied to genomic data by generalizing nucleotides into broader types based on their biochemical properties to satisfy 2 -anonymity ${ }^{88}$. Another countermeasure based on $k$-anonymity was proposed ${ }^{89}$ to thwart recent linkage attacks using signal profiles ${ }^{90}$ and raw data from functional genomics (for example, RNA sequences) ${ }^{89}$. Still, given the high dimensionality of genomic data, strategies based on generalization or randomization ${ }^{84}$ are unlikely to maintain the data at a level of detail that is useful for practical study. Thus, certain legal mechanisms, such as the HIPAA Expert Determination pathway, which we detail later on, tie the notion of de-identification to a re-identification risk assessment based on the 
capabilities of a reasonable data recipient ${ }^{91}$. For research, the utility (or usefulness) of genomic data should be maximized when subjecting it to a protection (or transformation) method. As such, Wan et al. demonstrated how to balance the tradeoff between utility and privacy using models based on game theory ${ }^{92,93}$.

Data aggregation. Although restricting access to data resources, such as the database of genotypes and phenotypes $(\mathrm{dbGaP})^{55}$, reduces privacy risks, it may also impede research advances. One potential alternative is a semi-trusted registration-based query system ${ }^{94}$ that processes queries internally and releases only summary results back to the users instead of releasing all individual-level data. For example, Beacon services (for example, the Beacon Network), popularized by the Global Alliance for Genomics and Health (GA4GH), let users query for only one type of information within genomic data sets ${ }^{95}$, namely the presence of alleles. Although a membership inference attack against Beacon services was demonstrated by Shringarpure and Bustamante ${ }^{96}$ in 2015 and enhanced later ${ }^{97,98}$, the effects of this attack can be mitigated by adding noise ${ }^{99,100}$, imposing query budgets ${ }^{99}$, adding relatives ${ }^{101}$ or strategically changing query responses for a subset of genetic variants ${ }^{102}$.

Data obfuscation. Obfuscating, or adding noise to, summary statistics based on a computational model, such as differential privacy (DP), has been used to counteract membership inference attacks ${ }^{103}$. However, the role of DP is limited in protecting GWAS and other data sets ${ }^{104,105}$ because a large amount of noise is required to provide protection ${ }^{27}$. Even if aggregate statistics are released with significant noise, membership and attribute information can still be inferred ${ }^{106}$. To preserve privacy, the resulting utility of the DP model is therefore often extremely ${ }^{\prime} w^{61}$. However, higher data utility could be achieved when assuming a weaker adversarial model ${ }^{107}$ or combining DP with modern cryptographic frameworks (for example, homomorphic encryption (HE), which we detail later on) ${ }^{108}$.

Synthetic data generation. Recently, researchers have proposed protecting anonymity by generating synthetic genomic data sets using deep learning models (for example, generative adversarial networks ${ }^{109,110}$ or restricted Boltzmann machines ${ }^{110}$ ). The generated data aim to maintain utility by replicating most of the characteristics of the source data and thus have the potential to become alternatives for many genomic databases that are not publicly available or have accessibility barriers.

A privacy protection model that publishes summary statistics about a data set while guaranteeing all potential attackers can learn virtually nothing more about an individual than they would learn if that person's record were absent from the data set.

Adversarial model

A model that characterizes attackers' behaviours and incentives with certain assumptions.
General Data Protection Regulation. International data privacy legislation is likely to alter the landscape of data privacy protection in genomics research around the world moving forward. The most notable example is the EU's GDPR, which took effect in 2018 and places restrictions on entities that handle the personal information of citizens of the EU, including genetic information ${ }^{12}$. The regulations grant data subjects access and deletion rights, impose security and breach notification requirements on entities that handle personal information, and place restrictions on the use and sharing of data without informed consent. Since the GDPR was enacted, there has been heated debate about its impact on the flow of data and hence the conduct of genomics research. Shabani and Marelli, for example, focus on the GDPR's recognition of the contextual nature of risk, and particularly the risk of re-identification, which they suggest can be ameliorated by compliance with codes of conduct or professional society guidance ${ }^{111}$. Mitchell et al. suggest that it may be necessary to have more stringent controls as well as to analyse data in place to avoid sharing ${ }^{112}$. In a subsequent news story, Mitchell also pointed out the complications posed by the emergence of identified ancestry databases ${ }^{113}$.

The United States has several laws that address the issue of identifiability, some of which have been in place for many years, and which differ in important ways both from each other and from the GDPR ${ }^{113}$.

United States: HIPAA. One of the most important laws governing patient care and biomedical research is the HIPAA and its Privacy Rule, which is limited in its oversight to data in the possession of three types of covered entities (that is, health-care providers, health plans and health-care clearinghouses) as well as the business associates of such entities ${ }^{114}$. HIPAA generally requires these entities to obtain patient authorization for uses and disclosures of protected health information outside of treatment, payment, and health-care operations and conveys access rights to individuals ${ }^{115}$.

However, the protections provided by HIPAA even within 'covered entities' contain numerous exceptions ${ }^{116}$. In particular, HIPAA does not require permission to use or disclose health information, including genomic information, if it has been de-identified through either one of two mechanisms that are colloquially referred to as 'Safe Harbour' and 'Expert Determination'. HIPAA defines de-identified data as follows: "Health information that does not identify an individual and with respect to which there is no reasonable basis to believe that the information can be used to identify an individual is not individually identifiable health information." The Safe Harbour approach requires the removal of an enumerated list of 18 explicit identifiers (for example, names, social security numbers) and quasi-identifiers (for example, date of birth and 5-digit ZIP code of residence) $)^{116}$ as well as an absence of actual knowledge that the remaining information could be used alone or in combination with other information to identify the individual. By contrast, the alternative Expert Determination pathway requires the application of statistical and/or computational mechanisms to show that the risk of 


\section{REVIEWS}

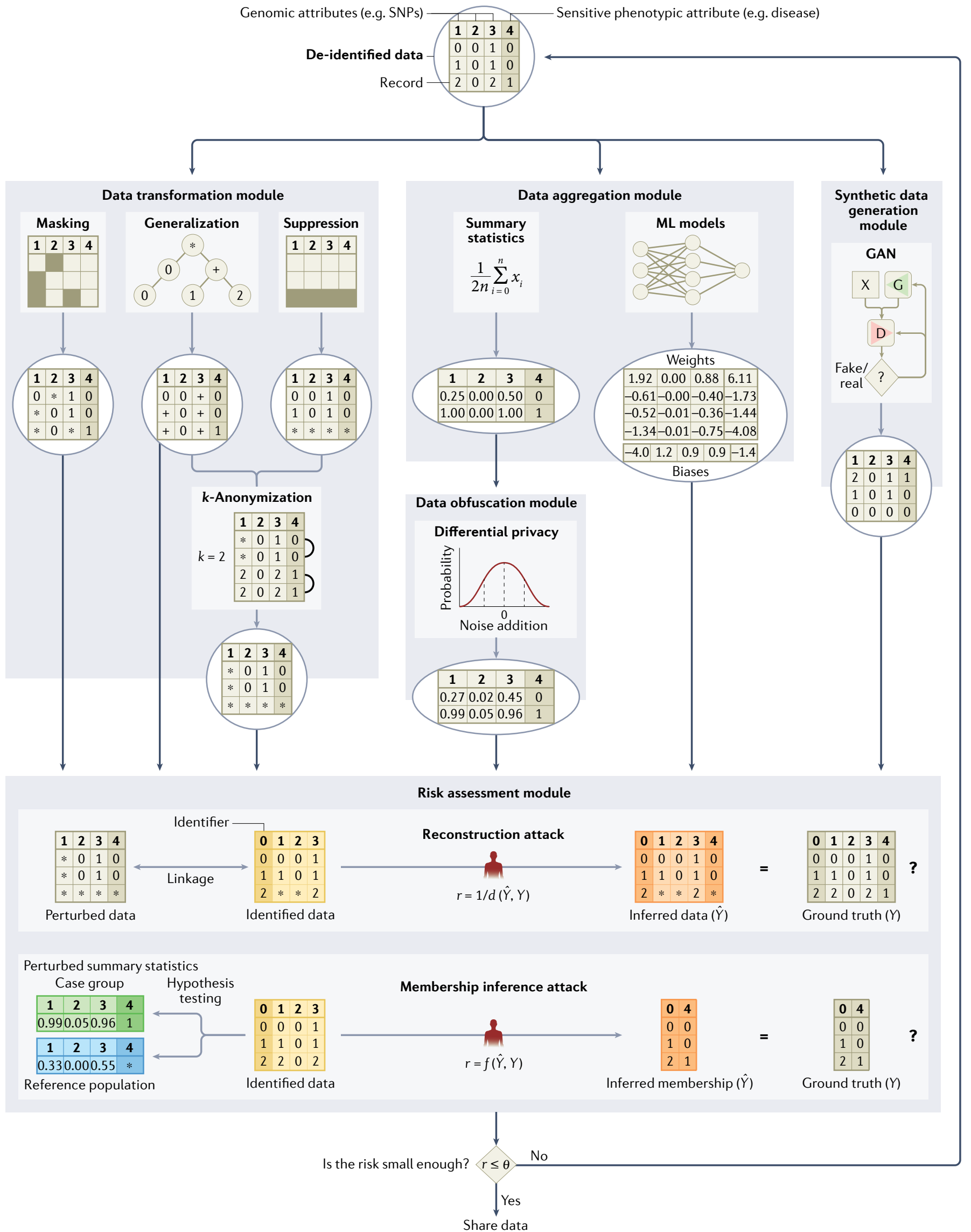

Data recipient 
4 Fig. 2 | Data perturbation approaches for privacy protection in genomic data sharing. Each module (or submodule) can work independently to protect data as shown by the corresponding data flow. In the transformation module, data can be masked ${ }^{93}$, generalized $^{88}$ and/or suppressed according to a privacy protection model (for example, $k$-anonymity $)^{87}$. In the aggregation module, data can be aggregated to summary statistics $^{81}$ or parameters in a machine learning $(\mathrm{ML})$ model $^{61}$. In the module of synthetic data generation, a synthetic data set can be generated using a generative adversarial network (GAN) $)^{110}$. In the obfuscation module, noise can be added to data using a privacy protection model (for example, differential privacy) ${ }^{103}$. All contents in each module (or submodule) are examples for illustration purposes only. In the example for the generalization submodule, the plus sign represents a generalization of values one and two for a genomic attribute. In the example for the submodule of summary statistics, the minor allele frequency for each single-nucleotide polymorphism (SNP) marker is computed for each group of individual records. ( $n$ represents the number of records in the group; $x_{i}$ represents the value of a genomic attribute for the $i^{\text {th }}$ record in a group, which is the number of minor alleles at a SNP position for a record in this example.) In the example for the submodule of ML models, the neural network with three layers has 21 parameters (that is, 16 weights and 5 biases) that need to be learned. In the example for the GAN submodule, $X$ represents the input data set, $\mathrm{G}$ represents the generator network and D represents the discriminator network. In the example for the reconstruction attack in the module of risk assessment ${ }^{91}$, the attacker tries to reconstruct the original data set by linkage and inference ${ }^{66}$, and the privacy risk is assessed by the data sharer using a distance function. In the example for the membership inference attack in the module of risk assessment ${ }^{92}$, the attacker tries to infer the membership of each targeted individual by hypothesis testing ${ }^{58}$, and the privacy risk is assessed by the data sharer using a function that measures the test's accuracy. The reconstruction attack and the membership inference attack are used here for illustration purposes only and could be replaced with any other attack (for example, a re-identification attack or a familial search attack) or some arbitrary combination of attacks. Data can be sequentially protected by multiple modules and submodules before the privacy risk is mitigated to an acceptable level and finally released. $r$ represents the privacy risk; $d$ represents the distance function; $f$ represents the function measures accuracy; $\theta$ represents the threshold for the privacy risk.
Much research that utilizes genetic data qualifies as minimal risk under the recently revised Common Rule and could therefore be eligible for expedited Institutional Review Board review ${ }^{122}$ and waiver of consent ${ }^{123}$. In addition, secondary research involving data that were initially collected for some other clinical or research purpose and has been transformed into a non-identified state (that is, data that have "been stripped of identifiers such that an investigator cannot readily ascertain a human subject's identity") ${ }^{124}$ is currently exempt from Common Rule regulations altogether, especially since a proposal to consider biospecimens and DNA data as identifiable per se was explicitly rejected when the Rule was revised in 2017. Thus, informed consent is not required for such research, a result that is generally much more permissive than the exceptions permitted under HIPAA. However, regulations governing identifiability may change in the future as federal departments and agencies were charged with formally re-examining the definition of 'identifiable private information' and 'identifiable biospecimen' over time, expecting that emerging technologies, such as whole-genome sequencing, may make genomic data more easily distinguishable.

Other legal issues in the United States. The courts in the United States, especially those at the federal level, have been reluctant to endow individuals with a right to control access to biospecimens or resulting data ${ }^{125-127}$ or to extend legal protections to discarded DNA ${ }^{22}$. Moreover, the Genetic Information Nondiscrimination Act of 2008 (GINA) ${ }^{128}$, which nominally prohibits geneticbased discrimination in the context of health insurance and employment, is limited in its scope, applying only to asymptomatic individuals and offers no protection regarding other types of insurance (for example, life and long-term disability). The Affordable Care Act and the Americans with Disabilities Act fill only some of these gaps ${ }^{6}$.

State laws. By contrast, over the years, several US state legislatures have enacted laws that convey additional rights or protections to individuals with respect to genetic information about them. For example, some states have deemed genetic information to be the property of the individual being tested and/or require informed consent for genetic testing ${ }^{129}$. States may also impose security requirements for genetic data or other health records, regulate the retention of biospecimens and data, or convey additional protections to research participants. States, most notably California ${ }^{130,131}$, Virginia $^{132}$ and Colorado ${ }^{133}$, have adopted broad data privacy legislation that provides people with much greater control over some uses of information about them with yet-uncertain implications for genomic information in a variety of settings, including research ${ }^{134}$. Other states, including Florida ${ }^{135}$ and New York ${ }^{136}$, are considering legislation as well. The highly influential Uniform Laws Commission, which proposes statutes for adoption, explicitly defined "genetic sequencing information" as sensitive and thus subject to special protections in its proposed Uniform Personal Data Protect Act approved in July 2021 by the Commission ${ }^{137}$. These proposed and 
a Homomorphic encryption

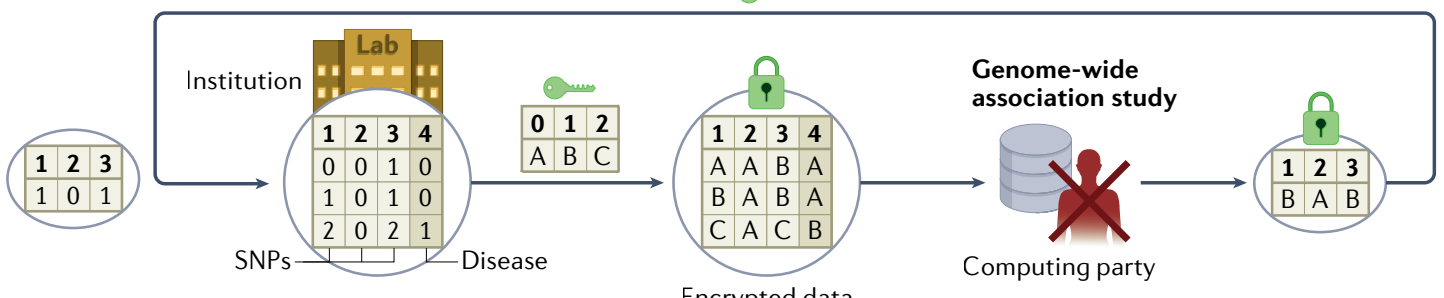

Encrypted data

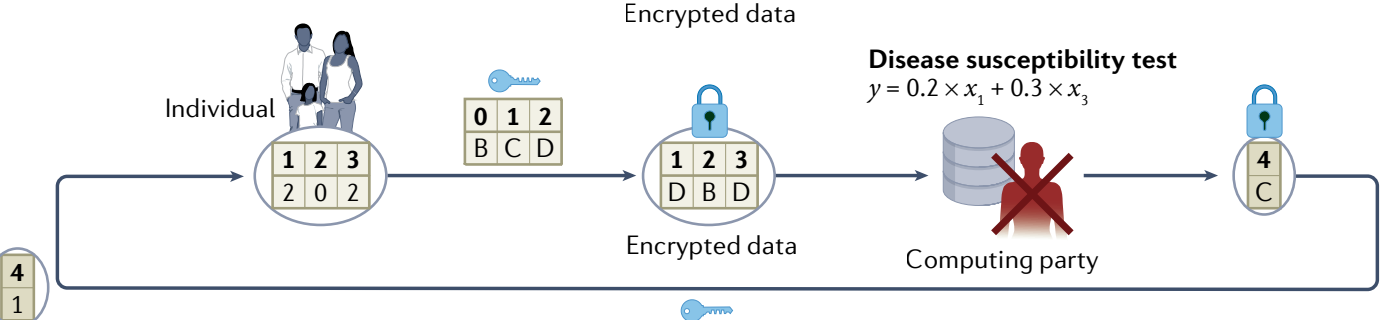

b Secure multiparty computation

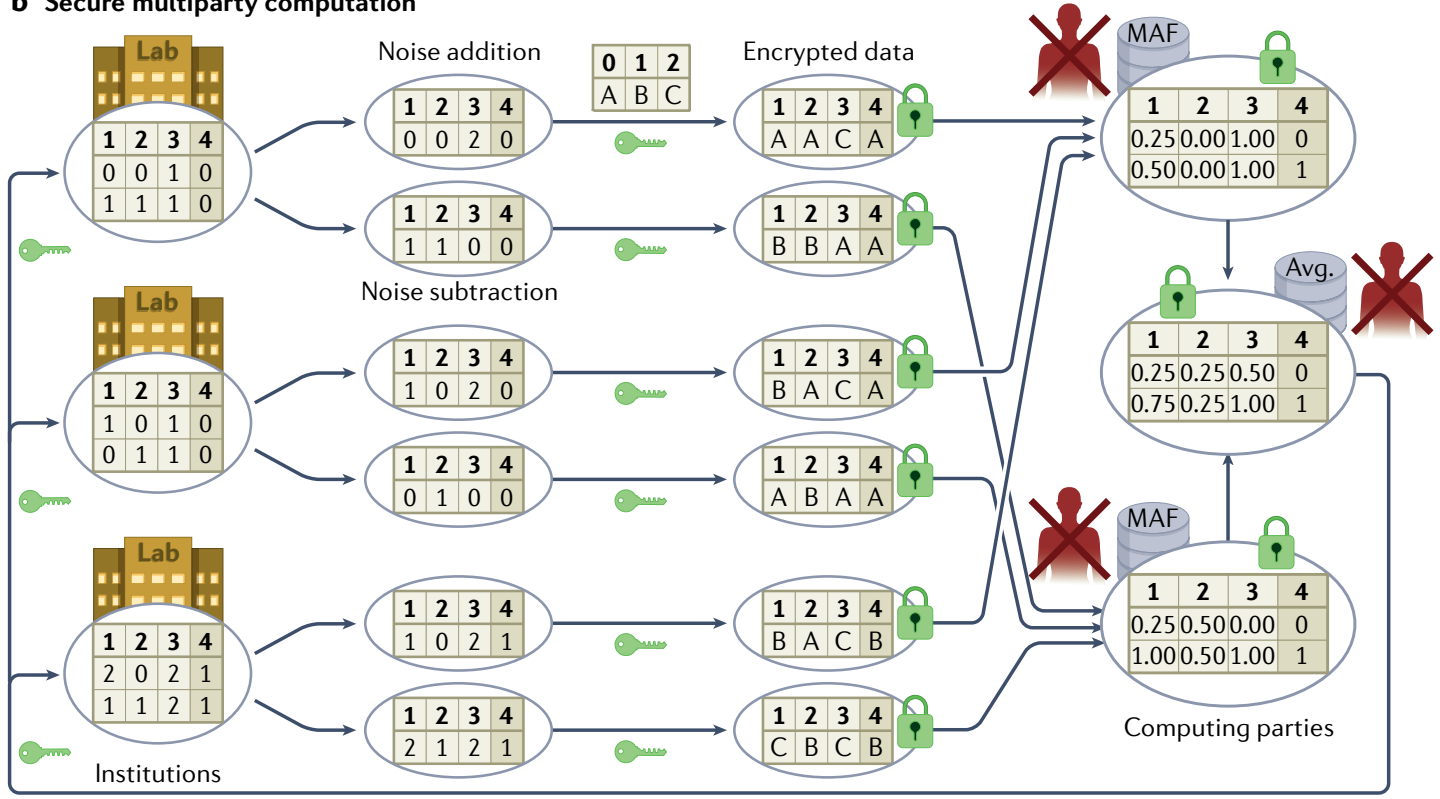

c Trusted execution environment
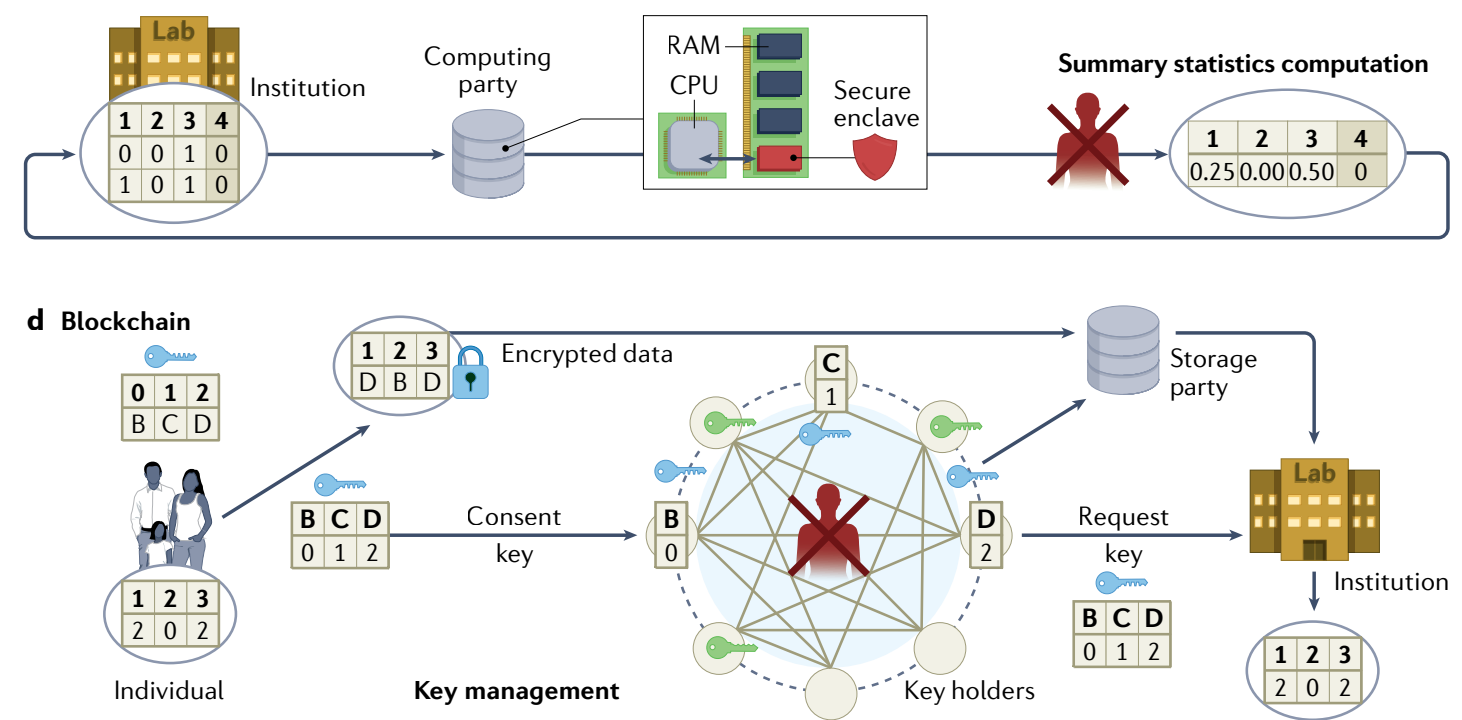
4 Fig. 3 | Cryptographic approaches for privacy protection in the use of genomic data. a | Homomorphic encryption enables computation by a third party on encrypted data without decrypting any specific record ${ }^{141}$. In this instance, it is applied to a genome-wide association study ${ }^{142}$ and a disease susceptibility test ${ }^{78} . \mathbf{b} \mid$ Secure multiparty computation enables multiple parties to jointly compute a function of their inputs without revealing inputs ${ }^{146}$. Here, three institutions share encrypted data to third parties for summary statistics (for example, minor allele frequency (MAF)) computing ${ }^{145} . \mathbf{c} \mid$ A trusted execution environment, such as Intel Software Guard Extensions (SGX) ${ }^{152}$, isolates the computation process in an encrypted enclave using central processing unit (CPU) support so that even malicious operating system software cannot see the enclave contents ${ }^{153}$. Here, an institution computes summary statistics (for example, MAF) in a secure enclave of a third party. $\mathbf{d} \mid$ A blockchain enables encrypted immutable records stored on a decentralized network ${ }^{161}$. Here, the individual manages the decryption key using a blockchain while sharing encrypted data with researchers ${ }^{32}$. Avg., average; RAM, random-access memory; SNP, single-nucleotide polymorphism.

Homomorphic encryption (HE). A form of encryption that permits computations on encrypted data without revealing the data to any of the parties involved in the cryptographic protocol.

\section{Secure multiparty}

computation

(SMC). A form of encryption that enables multiple parties to jointly compute a function of their inputs without revealing inputs.

Minor allele frequency The proportion of the second most common of two alleles at a genomic position in a population. An allele corresponds to one of two or more forms of genetic variant at a genetic position. An individual inherits two alleles for each genetic position, one from each parent.

Population stratification The systematic difference in allele frequencies between subpopulations of a collection of individuals. enacted laws commonly grant more access and correction rights to individuals and impose more restrictions on the use and sharing of personal information without informed consent and thus approach more closely the structure of the GDPR ${ }^{138}$. Nonetheless, the differences among these statutes themselves and in relation to current federal and international law will doubtless further complicate compliance.

\section{Genomic privacy in context Context matters}

The primary focus of this Review is addressing the complex ethical, legal and technical challenges that arise in protecting privacy in genomic research. Focusing solely on genomic research fails to take into account the potential impact on privacy of the increasing availability of such data in other settings. A wide variety of individuals and entities now collect, use and share genomic data at an unprecedented level. As a result, these data are becoming an increasingly viable resource for parties who might wish to exploit the data, including not only researchers, but also employers, insurers, law enforcement and other individuals ${ }^{33}$, many of whom have garnered much more media attention than those conducting biomedical investigations. Numerous studies suggest that some people are worried about where genomic data about them go and how they are used, potentially affecting them in ways they neither desire nor expect. In addition to more commonly explored fears of discrimination ${ }^{3}$, this information can also redefine family relationships, for example, by confirming or disproving paternity, locating previously unknown relatives, or identifying anonymous gamete donors ${ }^{139}$. These concerns about use and impact, generally couched in terms of desire for genetic privacy, may affect individuals' willingness to undergo clinical testing or to participate in research ${ }^{3,140}$. Such reluctance due to privacy concerns, in turn, may exacerbate existing health disparities and stifle scientific progress. Thus, when they design, conduct and discuss their research, investigators need to consider how genomic data are used and how the type of use affects whether or not the data are controlled outside the research setting as well.

\section{Research setting}

Technical protections. Researchers often use genomic data accompanied by an array of phenotypic and other information, which they may obtain from individuals directly, through health-care providers or from third parties such as DTC-GT companies. A researcher may also transfer data to third parties for computation or collaboration purposes. Many cryptographic tools can be deployed to protect such use of data from unauthorized access $^{29}$. FIGURE 3 illustrates four cryptographic protection approaches with examples in the context of genomic data use cases.

Specifically, FIG. 3a illustrates a use case in which an institution that lacks computing capability outsources a computation task (for example, GWAS) to a third party while keeping the data encrypted. Homomorphic encryption (HE) enables computation on encrypted data sets without ever decrypting any specific record and can be utilized when the computation of statistics (for example, counts $^{141}$, chi-square statistics ${ }^{142}$ and regression coefficients ${ }^{143}$ ) is outsourced to external data centres or public clouds ${ }^{144}$.

To generate statistically meaningful findings in the research setting, GWAS need many thousands of records that are often distributed among multiple repositories across various institutions, and even across jurisdictions. Secure multiparty computation (SMC), unlike HE, enables multiple parties to jointly compute a function of their inputs without revealing inputs ${ }^{28}$, as illustrated in FIG. 3b, in which three institutions jointly compute summary statistics (for example, minor allele frequency) over their private data sets. SMC enables the computation of GWAS statistics over distributed encrypted repositories without the local statistics being released ${ }^{145}$, and it can facilitate quality control and population stratification correction in large-scale GWAS ${ }^{146}$. SMC can also be applied to sequence matching in other settings ${ }^{147,148}$. Compared to federated learning, which enables multiple parties to jointly train ML models on genomic data sets over local statistics ${ }^{149}$, SMC guarantees a much higher security level at the cost of computationally expensive encryption operations. To reduce both the computation overhead and the communication burden, SMC can be combined with HE to support GWAS analyses among a large number (for example, 96) of parties ${ }^{150}$.

Cryptographic hardware can be leveraged to reduce the burden of computation (for example, secure count queries) $)^{151}$ on encrypted data using $\mathrm{HE}$ or $\mathrm{SMC}^{152}$. For example, a trusted execution environment based on Intel Software Guard Extensions (SGX) isolates the computation process in a protected enclave on one's computer ${ }^{153}$, as illustrated in FIG. $3 \mathrm{c}$, in which an institution outsources the task of computing summary statistics (for example, minor allele frequency) to a third party. Combining hardware (for example, Intel SGX) and algorithmic tools (for example, $\mathrm{HE}^{154}$ or sketching ${ }^{155}$ - a data summarization method) can enable users to perform secure GWAS analyses efficiently.

Blockchains can be adopted to incentivize genomic data sharing ${ }^{156}$ while protecting privacy ${ }^{32,157}$. For example, researchers have proposed to use blockchains to securely share GWAS data sets ${ }^{158}$ or parameters of ML models trained on genomic data sets ${ }^{159}$. FIGURE $3 d$ illustrates a distributed data sharing system, in which multiple independent parties hold shares of a split decryption key and maintain a blockchain that receives data access 
requests from researchers and consent from individual participants ${ }^{32}$. Combined with HE and SMC, blockchains can enable privacy-preserving analysis on genomic data in a personally controlled ${ }^{160}$ and transparent manner ${ }^{161}$. However, numerous practical challenges with blockchains remain, including scalability, efficiency and $\operatorname{cost}^{157}$.

Legal protections. Countries around the world have put in place provisions regarding the protection of human research participants, which typically address the need to weigh the risks and benefits to participants, particularly for those who are vulnerable, to assess the scientific merit of protocols, to protect privacy and confidentiality, and to define the role of oversight by research ethics committees and the role of informed consent ${ }^{162}$. Although the details differ across countries, the most recent version of the Declaration of Helsinki, the foundational document for international research ethics, generally requires consent only for "medical research using identifiable human Organizations of Medical Sciences addressed this issue in greater depth in Guideline 11 of its most recent report in 2016 on International Ethical Guidelines for Health-related Research Involving Humans ${ }^{164}$.

More generally, several international laws influence the ability to access or share genomic data. As noted above, the GDPR provides individuals with substantial control over data about them, typically requiring consent for use and often forbidding the transfer of data to countries whose data protections are not substantially compliant with the GDPR ${ }^{165}$. Citing several national and individual interests, China heavily regulates when human genomic data can leave the country and requires governmental approval ${ }^{166,167}$. India ${ }^{168}$ and many countries in Africa ${ }^{169}$ have similar practices.

The United States lacks an overarching national data privacy policy and does not typically impose limits on the export of genomic data ${ }^{170}$. Moreover, the legal protections afforded to genomic information shared with researchers depend heavily on the entity carrying out the research and the nature of the information (for example, whether it is shared in identifiable form or is instead converted into de-identified or aggregated data) as discussed above.

\section{Non-research settings}

In recent years, the use of genomic data in non-research settings has garnered an enormous amount of public attention and can have important implications for personal privacy.

Private set intersection A cryptographic technique that allows two parties to compute the intersection of their data without exposing their raw data to the other party.

Fuzzy encryption

In a fuzzy encryption scheme, the encrypted data can be decrypted by a set of similar keys

have undergone DTC-GT with companies that purport to provide personal information about a variety of issues, including health, ancestry, family relationships (for example, paternity), and lifestyle and wellness ${ }^{171-173}$. There are numerous media stories about how consumers use these data to reveal biological relationships, uses that elicit complex responses ${ }^{139}$, both positive and negative. Some people are pleased to find new relatives or to uncover their biological origins, whereas others are distressed by the results or by unwanted contact. material or data" 163 . The Council for International

There are, however, virtually no legal constraints on how consumers may use these data, although the legal consequences that may result from their actions could be considerable, including divorce and efforts to avoid support for children ${ }^{19}$.

The companies offering these services generally fall outside of the purview of the Common Rule and HIPAA (being neither federally funded nor a HIPAA-covered entity, respectively). Instead, the flow of genetic data in the DTC setting is governed largely by self-regulation and notice-and-choice in the form of privacy policies and terms of service ${ }^{172,173}$. Recent surveys of the privacy policies and terms of service of DTC-GT companies reveal tremendous variability across the industry, with many companies failing to meet best practices and guidelines concerning privacy, secondary uses of genetic information, and sharing of data with third parties ${ }^{172-174}$.

Although the industry has largely been left to self-regulate, federal agencies have played a limited role in shaping policy with respect to DTC-GT. For example, the US Food and Drug Administration has exercised oversight over a narrow category of DTC health-related tests, although the trend has been to allow these tests to enter the market with little resistance ${ }^{175}$. The baseline of protection is provided by the Federal Trade Commission, which has the authority to police unfair and deceptive activities across all areas of commerce. Perhaps hindered by its broad mandate and limited resources, the agency to date has only intervened in the DTC-GT space in one case of particularly egregious conduct (that is, unsubstantiated health claims coupled with a lack of security of consumer personal information, including genetic data $)^{176}$. Instead, the agency has chosen to embrace self-regulation, largely limiting its involvement to the issuance of consumer-facing bulletins ${ }^{177,178}$ about the implications of genetic testing and broad guidelines for companies offering DTC-GT in the form of a blog post ${ }^{179}$. For those who are interested, numerous technical strategies exist to permit two users to match genome sequences without disclosing their genomes by using $\mathrm{HE}^{180}$, private set intersection protocols ${ }^{181}$ or fuzzy encryption ${ }^{182}$, thereby providing additional privacy protections.

Importantly, millions of people have downloaded their results from DTC-GT and posted them on thirdparty databases to facilitate finding relatives or to obtain health-related interpretations. These sites are rarely subject to any type of regulation beyond what they specify in their terms of service ${ }^{173}$. Moreover, these sites reserve the right to change their practices, which may occur as a response to public pressure, but may also be due to changes in business operations. These are the data that facilitate forensic use and are likely to pose the greatest potential for re-identification of genomic data.
Forensic setting. Law enforcement looms large in public opinion about genetic data since it may seek to access genetic information, an issue that has gained intense interest in the wake of high-profile cold cases that were ultimately solved using such information ${ }^{183}$. Over the years, there has also been an effort to expand government-run forensic databases at the federal, 
state and local levels ${ }^{184}$. The FBI currently maintains a nationwide database, the Combined DNA Index System (CODIS), that contains the genetic profiles of over 20 million individuals ${ }^{185}$ who have been either arrested or convicted of a crime as well as over 1 million forensic profiles derived from crime scenes ${ }^{186}$.

Law enforcement may also seek to compel the disclosure of genetic information held by an individual or an entity such as a health-care provider, DTC-GT company or researcher. A subpoena is generally all that is required to compel disclosure of genetic information in a patient's electronic medical record under HIPAA $^{187}$. Genetic data held by researchers may be shielded by government-issued Certificates of Confidentiality, which purport to assure participants that such data are immune from court orders and outside the reach of law enforcement, but these are issued by default only to research funded by the NIH and other agencies within HHS and may not protect research data that are placed in participants' electronic health records as well as disclosures required by federal, state and local laws ${ }^{188,189}$.

Furthermore, law enforcement may also seek to exploit public databases or utilize the services of a DTC-GT company for forensic genealogy purposes in FGG/IGG. To date, law enforcement in the United States has largely focused its efforts on publicly accessible databases (for example, GEDmatch) ${ }^{183}$ and private databases held by companies that voluntarily cooperate (for example, FamilyTreeDNA) ${ }^{190}$. For example, law enforcement generated leads in dozens of cold cases by uploading genetic profiles derived from crime scenes to GEDmatch, a public database where individuals can upload their DTC-GT data to learn about where their forebears came from and to locate potential genetic relatives. Similarly, FamilyTreeDNA provides law enforcement access to a version of their Family Finder service, which, like GEDmatch, allows consumers to upload DTC-GT data to locate potential relatives.

In response to public privacy concerns, both GEDmatch and FamilyTreeDNA changed their policies to either require consumers to opt in for their genetic information to be used for law enforcement matching or provide an opportunity to opt out, rather than allowing such searches by default ${ }^{68}$. This change dramatically reduced the pool of users available to law enforcement, leading them to seek court orders to explore the entire databases of GEDmatch and Ancestry.com, respectively ${ }^{187,191}$.

In 2019, the US Department of Justice released an interim policy statement designed to signal its intentions regarding privacy and the use of FGG/IGG ${ }^{192}$. The interim guidelines, which have not been updated since, impose several limitations on federal law enforcement agencies, such as limiting these searches to investigations of serious violent crimes (ill-defined in the guidelines), requirements barring deception on the part of law enforcement when utilizing a DTC service, and requirements that the company seek informed consent from consumers surrounding their cooperation with law enforcement ${ }^{192}$. At least one local district attorney's office has developed, and voluntarily adopted, similar guidelines ${ }^{193}$. Given the recent emergence of these tools, it is perhaps of little surprise that legal regimes are evolving in different ways across the country and around the world ${ }^{194,195}$.

At the same time, there has been limited research into techniques to mitigate kinship privacy risks ${ }^{196}$ stemming from the familial genomic searches at the core of FGG/IGG. One general approach is to optimize the choice of SNPs that are masked to minimize the likelihood of successful inference based on relatives' genomic information ${ }^{196}$, but little follow-up work has been done on this topic.

\section{Conclusions}

As this Review shows, providing appropriate levels of privacy for genomic data will require a combination of technical and societal solutions that consider the context in which the data are applied. Yet, there are challenges to achieving such goals. From a technical perspective, for instance, it is non-trivial to move from privacy-enhancing and security-enhancing technologies that are communicated in a paper or tested in a small pilot study to a full-fledged enterprise-scale solution. This challenge is not unique to genomic data as it is a dilemma for data more generally and for the application domains in which data are applied. In addition, one of the core problems is that it is difficult to build privacy into infrastructure after it has been deployed. Rather, privacy-by-design ${ }^{197}$, whereby the principles of privacy are articulated at the outset of a project or the point at which data are created and are tailored to the environment to which they are shared, may provide a more systematic and sustainable approach to genomic data protection. However, even if the principles are clearly articulated, there is no guarantee that the technology will support privacy in the long term. For instance, HE, one of the technologies emerging for secure computation over genomic data, is constantly evolving. This may make it difficult to compare genomic data encrypted at one point in time with genomic data created under a more recent version of the technology. Moreover, encryption technologies are not necessarily ideal for long-term management of data ${ }^{198}$, especially since new computing technologies, such as cheap cloud computing and quantum computing, might make it extremely cheap to crack such encryptions.

Beyond technology, numerous social factors, which inevitably involve tradeoffs between protection and utility, further complicate efforts to protect genomic privacy. Countries, for example, vary dramatically in how much control individuals have over how genomic data about them are used. Some provide individuals granular control while others permit use without consent in many settings, albeit often with stringent security protections. More dramatic is the impact of the growing number of people who post identified genomic data about themselves so that they can find relatives or connect with people who have similar conditions or history. Yet, people who share identified data about themselves increase the potential to re-identify other data about them. In addition, they also reveal information about their relatives, some of whom might have preferred more privacy. These consumer-created databases, unlike medical and 
research records, frequently have few limitations on use by third parties as has been illustrated by the growth of forensic genealogy. Deciding how to make tradeoffs between protection and use across the entire ecology of genomic data flows requires consideration of both the value of these interests as well as practicable mechanisms of control.

Pressure is growing to protect genomic privacy with security-enhancing technologies and legal regimes for use of genomic data. Nonetheless, it seems clear that simply giving individuals granular control over genomic data that pertain to them, by itself, while attractive to some, risks reifying an unwarranted fear of genomics and is likely to disrupt a wide array of advances in ways that almost surely do not align with the public's preferences. What may well be needed is a combination of notice and some choice, accountable oversight of uses, and real penalties - both economic and reputational - for inflicting harm on individuals and groups. An additional requirement could be the creation of secure databases for specific purposes (for example, research versus ancestry versus criminal justice) with privacy-protecting tools and individual choice for inclusion that is appropriate for each, which can take the form of law $^{39}$ as well as private ordering using tools such as data use agreements ${ }^{36}$. Creating such a complex system will not be elegant and will need to evolve in response to how new laws and privacy-enhancing technologies affect individuals and groups, but simple solutions will not suffice either to protect people and populations from harm or to advance knowledge to improve health.

Published online 4 March 2022
1. Garrison, N. A. Genomic justice for Native Americans: impact of the Havasupai case on genetic research Sci. Technol. Hum. Values 38, 201-223 (2013).

2. Spector-Bagdady, K. et al. "My research is their business, but I'm not their business": patient and clinician perspectives on commercialization of precision oncology data. Oncologist 25, 620-626 (2020)

Clayton, E. W., Halverson, C. M., Sathe, N. A. ¿ Malin, B. A. A systematic literature review of individuals' perspectives on privacy and genetic information in the United States. PLOS ONE 13 e0204417 (2018).

This work provides a comprehensive overview of the literature surrounding individual's perspectives on genetic privacy in the United States.

4. Doe, G. With genetic testing, I gave my parents the gift of divorce. Vox https://www.vox.com/2014/9/9/ 5975653/with-genetic-testing-i-gave-my-parentsthe-gift-of-divorce-23andme (2014).

5. Copeland, L. The Lost Family: How DNA Testing is Upending Who We Are (Abrams, 2020).

6. Clayton, E. W. Why the Americans With Disabilities Act matters for genetics. JAMA 313, 2225-2226 (2015).

7. McKibbin, K. J., Malin, B. A. \& Clayton, E. W. Protecting research data of publicly revealing participants. J. Law Biosci. 8, Isab028 (2021).

8. Solove, D. J. A taxonomy of privacy. Univ. Pa. Law Rev. 154, 477-564 (2006).

9. Niemiec, E. \& Howard, H. C. Ethical issues in consumer genome sequencing: use of consumers' samples and data. Appl. Transl. Genom. 8, 23-30 (2016).

10. Obar, J. A. \& Oeldorf-Hirsh, A. The biggest lie on the Internet: ignoring the privacy policies and terms of service policies of social networking services. Inf. Commun. Soc. 23, 128-147 (2020).

11. Geier, C., Adams, R. B., Mitchell, K. M. \& Holtz, B. Informed consent for online research-is anybody reading?: assessing comprehension and individual differences in readings of digital consent forms. J. Empir. Res. Hum. Res. Ethics 16, 154-164 (2021).

12. The European Parliament and The Council Of The European Union. General Data Protection Regulation, Regulation (EU) 2016/679. Official J. Eur. Union https://eur-lex.europa.eu/eli/reg/2016/679/oj (2016).

13. Code of Federal Regulations. Title 45, section 164.502 Uses and disclosures of protected health information: general rules (d)(2). eCFR https://www.ecfr.gov/current/ title-45/subtitle-A/subchapter-C/part-164/subpart-E/ section-164.502\#p-164.502(d)(2) (2021).

14. Code of Federal Regulations. Title 45, section 164.502: Other requirements relating to uses and disclosures of protected health information (a). eCFR https://www.ecfr.gov/current/title-45/subtitle-A/ subchapter-C/part-164/subpart-E/section-164.514\#p164.514(a) (2021)

15. Code of Federal Regulations. Title 45, section 164.502 Other requirements relating to uses and disclosures of protected health information (b). eCFR https://www.ecfr. gov/current/title-45/subtitle-A/subchapter-C/part-164/ subpart-E/section-164.514\#p-164.514(b) (2021)

16. Code of Federal Regulations. Title 45, part 46: Protection of human subjects. eCFR https:// www.ecfr.gov/current/title-45/subtitle-A/subchapter-A/ part-46 (2018)
17. Brandeis, L. \& Warren, S. The right to privacy. Harv. Law Rev. 4, 193-220 (1890).

18. Burke, W. et al. Recommendations for returning genomic incidental findings? We need to talk! Genet. Med. 15, 854-859 (2013).

19. Jarvik, G. P. et al. Return of genomic results to research participants: the floor, the ceiling, and the choices in between. Am. J. Hum. Genet. 94, 818-826 (2014).

20. Hazel, J. W. et al. Direct-to-consumer genetic testing: prospective users' attitudes toward information about ancestry and biological relationships. PLOS ONE 16, e0260340 (2021).

21. Garner, S. A \& Kim, J. The privacy risks of direct-toconsumer genetic testing: a case study of 23 andMe and Ancestry. Wash. Univ. Law Rev. 96, 1219 (2019).

22. Clayton, E. W., Evans, B. J., Hazel, J. W. \&

Rothstein, M. A. The law of genetic privacy: applications, implications, and limitations. J. Law Biosci. 6, 1-36 (2019).

This work provides a comprehensive overview of the legal landscape surrounding genetic privacy in the United States.

23. Kaye, J. The tension between data sharing and the protection of privacy in genomics research. Annu. Rev. Genomics Hum. Genet. 13, 415-431 (2012).

24. Knoppers, B. M. \& Thorogood, A. M. Ethics and big data in health. Curr. Opin. Syst. Biol. 4, 53-57 (2017).

25. Biller-Andorno, N., Capron, A. M. \& Elger, B. Ethical Issues in Governing Biobanks: Global Perspectives (Routledge, 2016).

26. Malin, B. A. An evaluation of the current state of genomic data privacy protection technology and a roadmap for the future. J. Am. Med. Inform. Assoc. 12, 28-34 (2005)

27. Erlich, Y. \& Narayanan, A. Routes for breaching and protecting genetic privacy. Nat. Rev. Genet. 15 , 409-421 (2014).

This work provides a comprehensive overview of the possible and plausible attacks against genetic privacy and their technical countermeasures.

28. Naveed, M. et al. Privacy in the genomic era. ACM Comput. Surv. 48, 1-44 (2015).

29. Wang, S. et al. Genome privacy: challenges, technical approaches to mitigate risk, and ethical considerations in the United States. Ann. NY Acad. Sci. 1387, 73-83 (2017).

30. Arellano, A. M., Dai, W., Wang S., Jiang, X. \& Ohno-Machado, L. Privacy policy and technology in biomedical data science. Annu. Rev. Biomed. Data Sci. 1, 115-129 (2018)

31. Mittos, A., Malin, B. \& De Cristofaro, E. Systematizing genome privacy research: a privacy-enhancing technologies perspective. Proc. Priv. Enh. Technol. 2019, 87-107 (2019).

32. Grishin, D., Obbad, K. $₫$ Church, G. M. Data privacy in the age of personal genomics. Nat. Biotechnol. 37, 1115-1117 (2019)

33. Bonomi, L., Huang, Y. ¿ Ohno-Machado, L. Privacy challenges and research opportunities for genomic data sharing. Nat. Genet. 52, 646-654 (2020).

34. Ram, N. Genetic privacy after Carpenter. Va. Law Rev. 105, 1357-1425 (2019).
35. Noordyke, M. US state comprehensive privacy law comparison. IAPP https://iapp.org/news/a/ us-state-comprehensive-privacy-law-comparison/ (2019).

36. Hazel, J. W. \& Slobogin, C. "A world of difference"? Law enforcement, genetic data, and the fourth amendment. Duke Law J. 70, 705-774 (2020).

37. Wheeland, D. G. Final NIH genomic data sharing policy. Fed. Regist. 79, 51345-51354 (2014).

38. Rothstein, M. A. Informed consent for secondary research under the new NIH data sharing policy. J. Law Med. Ethics 49, 489-494 (2021).

39. Hazel, J. W., Clayton, E. W., Malin, B. A. \& Slobogin, C. Is it time for a universal genetic forensic database? Science 362, 898-900 (2018).

40. Zielinski, D. \& Erlich, Y. Genetic privacy in the post-COVID world. Science 371, 566-567 (2021).

41. Shelton, J. F. et al. Trans-ancestry analysis reveals genetic and nongenetic associations with COVID-19 susceptibility and severity. Nat. Genet. 53, 801-808 (2021).

42. Malin, B. \& Sweeney, L. How (not) to protect genomic data privacy in a distributed network: using trail re-identification to evaluate and design anonymity protection systems. J. Biomed. Inform. 37, 179-192 (2004).

43. Kayser, M. \& de Knijff, P. Improving human forensics through advances in genetics, genomics and molecular biology. Nat. Rev. Genet. 12, 179-192 (2011).

44. Lippert, C. et al. Identification of individuals by trait prediction using whole-genome sequencing data. Proc. Natl Acad. Sci. USA 114, 10166-10171 (2017).

45. Harmanci, A. \& Gerstein, M. Quantification of private information leakage from phenotype-genotype data: linking attacks. Nat. Methods 13, 251-256 (2016).

46. Humbert, M., Huguenin, K., Hugonot, J., Ayday, E. $\&$ Hubaux, J.-P. De-anonymizing genomic databases using phenotypic traits. Proc. Priv. Enh. Technol. 2015, 99-114 (2015).

47. Venkatesaramani, R., Malin, B. A. \& Vorobeychik, Y Re-identification of individuals in genomic datasets using public face images. Sci. Adv. 7, eabg3296 (2021)

48. Sero, D. et al. Facial recognition from DNA using face-to-DNA classifiers. Nat Commun. 10, 2557 (2019).

49. Erlich, Y. Major flaws in "Identification of individuals by trait prediction using whole-genome sequencing data". Preprint at bioRxiv https://doi.org/10.1101/185330 (2017).

50. Lippert, C et al No major flaws in "Identification of individuals by trait prediction using whole-genome sequencing data". Preprint at bioRxiv https://doi.org/ 10.1101/187542 (2017).

51. Malin, B. Re-identification of familial database records. AMIA Annu. Symp. Proc. 2006, 524-528 (2006).

52. Ball, M. P. et al. Harvard Personal Genome Project: lessons from participatory public research. Genome Med. 6, 10 (2014).

53. Sweeney, L., Abu, A. \& Winn, J. Identifying participants in the personal genome project by name (a re-identification experiment). Preprint at arXiv https://arxiv.org/abs/1304.7605 (2013).

54. Gymrek, M., McGuire, A. L., Golan, D., Halperin, E. $\&$ Erlich, Y. Identifying personal genomes by surname inference. Science 339, 321-324 (2013). 
55. Mailman, M. D. et al. The NCBI dbGaP database of genotypes and phenotypes. Nat. Genet. 39, 1181-1186 (2007)

56. Homer, N. et al. Resolving individuals contributing trace amounts of DNA to highly complex mixtures using high-density SNP genotyping microarrays. PLoS Genet. 4, e1000167 (2008).

57. Braun, R., Rowe, W., Schaefer, C., Zhang, J. \& Buetow, K. Needles in the haystack: identifying individuals present in pooled genomic data. PLoS Genet. 5, e 1000668 (2009).

58. Sankararaman, S., Obozinski, G., Jordan, M. I. \& Halperin, E. Genomic privacy and limits of individual detection in a pool. Nat. Genet. $\mathbf{4 1}$ 965-967 (2009)

59. Wang, R., Li, Y. F., Wang, X., Tang, H. \& Zhou, X. Learning your identity and disease from research papers: information leaks in genome wide association study. Proc. 16th ACM Conf. Comput. Commun. Secur. 2009, 534-544 (2009)

60. Im, H. K., Gamazon, E. R., Nicolae, D. L. \& Cox, N. J. On sharing quantitative trait GWAS results in an era of multiple-omics data and the limits of genomic privacy. Am. J. Hum. Genet. 90, 591-598 (2012).

61. Fredrikson, M. et al. Privacy in pharmacogenetics: an end-to-end case study of personalized warfarin dosing. Proc. 23rd USENIX Secur. Symp. 2014, 17-32 (2014).

62. Lumley, T. \& Rice, K. Potential for revealing individuallevel information in genome-wide association studies. JAMA 303, 659-660 (2010)

63. Humbert, M., Ayday, E., Hubaux, J.-P. \& Telenti, A Addressing the concerns of the Lacks family: quantification of kin genomic privacy. Proc. 2013 ACM Conf. Comput. Commun. Secur. 2013, 1141-1152 (2013).

64. Kong, A. et al. Detection of sharing by descent, longrange phasing and haplotype imputation. Nat. Genet. 40, 1068-1075 (2008).

65. Humbert, M., Ayday, E., Hubaux, J.-P. \& Telenti, A. Quantifying interdependent risks in genomic privacy. ACM Trans. Priv. Secur. 20, 3 (2017).

66. Deznabi, I., Mobayen, M., Jafari, N., Tastan, O. $\&$ Ayday, E. An inference attack on genomic data using kinship, complex correlations, and phenotype information. IEEE/ACM Trans. Comput. Biol. Bioinform 15, 1333-1343 (2018)

67. Callaway, E. Supercharged crime-scene DNA analysis sparks privacy concerns. Nature 562, 315-316 (2018)

68. Aldhous, P. This genealogy database helped solve dozens of crimes. But its new privacy rules will restrict access by cops. BuzzFeed News https:// www.buzzfeednews.com/article/peteraldhous/ this-genealogy-database-helped-solve-dozens-ofcrimes-but (2019).

69. Wood, A. DNA, genealogy led to arrest in series of rapes. Journal Inquirer https://web.archive.org/web/ 20220208235101/https://www.journalinquirer.com/ newsletters/dna-genealogy-led-to-arrest-in-series-ofrapes/article_27b25296-ab2d-11 ea-8b3e472861 ca42e0.html (2020).

70. Zhang, S. How a tiny website became the police's go-to genealogy database. The Atlantic https:// www.theatlantic.com/science/archive/2018/06/ gedmatch-police-genealogy-database/561695/ (2018)

71. Murphy, H. Why a data breach at a genealogy site has privacy experts worried. New York Times https:/ www.nytimes.com/2020/08/01/technology/ gedmatch-breach-privacy.html (2020).

72. Erlich, Y., Shor, T., Pe'er, I. \& Carmi, S. Identity inference of genomic data using long-range familial searches. Science 362, 690-694 (2018).

73. Kim, J., Edge, M. D., Algee-Hewitt, B. F. B., Li, J. Z $\&$ Rosenberg, N. A. Statistical detection of relatives typed with disjoint forensic and biomedical loci. Cell 175, 848-858 (2018).

74. Edge, M. D. \& Coop, G. Attacks on genetic privacy via uploads to genealogical databases. eLife $\mathbf{9}$, e51810 (2020).

75. Ney, P., Ceze, L. \& Kohno, T. Genotype extraction and false relative attacks: security risks to third-party genetic genealogy services beyond identity inference. Proc. Netw. Distrib. Syst. Secur. Symp. https://doi.org 10.14722/ndss.2020.23049 (2020).

76. Huang, Z., Ayday, E., Fellay, J., Hubaux, J.-P. ¿ Juels, A. Genoguard: protecting genomic data against brute-force attacks. Proc. 2015 IEEE Symp. Secur. Priv. 2015, 447-462 (2015).

77. Huang, Z. et al. A privacy-preserving solution for compressed storage and selective retrieval of genomic data. Genome Res. 26, 1687-1696 (2016).

78. Ayday, E., Raisaro, J. L., Hubaux, J.-P. \& Rougemont, J. Protecting and evaluating genomic privacy in medical tests and personalized medicine. Proc. 12th ACM Workshop Priv. Electron. Soc. 2013 95-106 (2013).

This is the first study to use homomorphic encryption for privacy-preserving clinical genetic testing.

79. Naveed, M. et al. Controlled functional encryption. Proc. 21st ACM Conf. Comput. Commun. Secur. 2014, 1280-1291 (2014).

80. Rodriguez, L. L., Brooks, L. D., Greenberg, J. H. \& Green, E. D. The complexities of genomic identifiability. Science 339, 275-276 (2013).

81. Zerhouni, E. A. \& Nabel, E. G. Protecting aggregate genomic data. Science 322, 44-44 (2008).

82. Craig, D. W. et al. Assessing and managing risk when sharing aggregate genetic variant data. Nat. Rev. Genet 12, 730-736 (2011).

83. Shi, X. \& Wu, X. An overview of human genetic privacy. Ann. NY Acad. Sci. 1387, 61-72 (2017).

84. Lin, Z., Owen, A. B. \& Altman, R. B. Genomic research and human subject privacy. Science 305, 183-183 (2004).

85. Edge, M. D., Algee-Hewitt, B. F. B., Pemberton, T. J. Li, J. Z. \& Rosenberg, N. A. Linkage disequilibrium matches forensic genetic records to disjoint genomic marker sets. Proc. Natl Acad. Sci. USA 114, 5671-5676 (2017).

86. Marchini, J. \& Howie, B. Genotype imputation for genome-wide association studies. Nat. Rev. Genet. 11 499-511 (2010)

87. Sweeney, L. $k$-anonymity: a model for protecting privacy. Int. J. Uncertain. Fuzz. 10, 557-570 (2002).

88. Malin, B. A. Protecting genomic sequence anonymity with generalization lattices. Methods Inf. Med. 44 , 687-692 (2005)

\section{This pioneering work shows the use of data} perturbation for genomic data privacy.

89. Gursoy, G et al. Data sanitization to reduce private information leakage from functional genomics. Cell 183, 905-917 (2020).

90. Harmanci, A. \& Gerstein, M. Analysis of sensitive information leakage in functional genomics signal profiles through genomic deletions. Nat. Commun. 9 2453 (2018)

91. Wan, Z. et al. A game theoretic framework for analyzing re-identification risk. PLOS ONE 10 e0120592 (2015)

92. Wan, Z. et al. Expanding access to large-scale genomic data while promoting privacy: a game theoretic approach. Am. J. Hum. Genet. 100 316-322 (2017)

This work maps a membership inference attack into a game theoretic framework and demonstrates ways by which optimal protection can be achieved.

93. Wan, Z. et al. Using game theory to thwart multistage privacy intrusions when sharing data. Sci. Adv. 7, eabe9986 (2021).

94. Dyke, S. O. M. et al. Registered access: authorizing data access. Eur. J. Hum. Genet. 26, 1721-1731 (2018).

95. Fiume, M. et al. Federated discovery and sharing of genomic data using beacons. Nat. Biotechnol. 37 220-224 (2019).

96. Shringarpure, S. S. \& Bustamante, C. D. Privacy risks from genomic data-sharing beacons. Am. J. Hum. Genet. 97, 631-646 (2015).

97. von Thenen, N., Ayday, E. \& Cicek, A. E. Re-identification of individuals in genomic data-sharing beacons via allele inference. Bioinformatics 35, 365-371 (2019).

98. Ayoz, K., Ayday, E. \& Cicek, A. E. Genome reconstruction attacks against genomic data-sharing beacons. Proc. Priv. Enh. Technol. 2021, 28-48 (2021).

99. Raisaro, J. L. et al. Addressing Beacon re-identification attacks: quantification and mitigation of privacy risks. J. Am. Med. Inform. Assoc. 24, 799-805 (2017)

100. Cho, H., Simmons, S., Kim, R. \& Berger, B. Privacy-preserving biomedical database queries with optimal privacy-utility trade-offs. Cell Syst. 10, 408-416 (2020)

101. Ayoz, K., Aysen, M., Ayday, E. \& Cicek, A. E. The effect of kinship in re-identification attacks against genomic data sharing beacons. Bioinformatics 36, i903-i910 (2020).

102. Wan, Z., Vorobeychik, Y., Kantarcioglu, M. \& Malin, B. Controlling the signal: practical privacy protection of genomic data sharing through Beacon services. BMC Med. Genomics 10, 39 (2017).
103. Uhlerop, C., Slavkovic, A. \& Fienberg, S. E. Privacypreserving data sharing for genome-wide association studies. J. Priv. Confid. 5, 137-166 (2013). This is the first study to use differential privacy for privacy-preserving GWAS data sharing.

104. Johnson, A. \& Shmatikov, V. Privacy-preserving data exploration in genome-wide association studies. Proc. 19th ACM SIGKDD Int. Conf. Knowl. Discov. Data Min. 2013, 1079-1087 (2013).

105. Simmons, S., Sahinalp, C. \& Berger, B. Enabling privacy-preserving GWASs in heterogeneous human populations. Cell Syst. 3, 54-61 (2016)

106. Almadhoun, N., Ayday, E. \& Ulusoy, O. Inference attacks against differentially private query results from genomic datasets including dependent tuples. Bioinformatics 36, i136-i145 (2020).

107. Tramèr, F., Huang, Z., Hubaux, J.-P. \& Ayday, E. Differential privacy with bounded priors: reconciling utility and privacy in genome-wide association studies Proc. 22nd ACM Conf. Comput. Commun. Secur. 2015, 1286-1297 (2015).

108. Raisaro, J. L. et al. Protecting privacy and security of genomic data in $\mathrm{i} 2 \mathrm{~b} 2$ with homomorphic encryption and differential privacy. IEEE/ACM Trans. Comput. Biol. Bioinform. 15, 1413-1426 (2018)

109. Bae, H., Jung, D., Choi, H.-S. \& Yoon, S. AnomiGAN: generative adversarial networks for anonymizing private medical data. Proc. 25th Pac. Symp. Biocomput. 2020 563-574 (2019).

110. Yelmen, B. et al. Creating artificial human genomes using generative neural networks. PLoS Genet. 17 e1009303 (2021)

111. Shabani, M. \& Marelli, L. Re-identifiability of genomic data and the GDPR: assessing the re-identifiability of genomic data in light of the EU General Data Protection Regulation. EMBO Rep. 20, e48316 (2019).

112. Michell, C., Ordish, J., Johnson, E., Bridgen, T. \& Hall, A. The GDPR and Genomic Data-the Impact of the GDPR and DPA 2018 on Genomic Healthcare and Research (PHG Foundation, 2020).

113. Petrone, J. Europe's genomics community wrestling with uncertainty presented by privacy legislation. genomeweb https://www.genomeweb.com/informatics/ europes-genomics-community-wrestling-uncertaintypresented-privacy-legislation (2021).

114. Code of Federal Regulations. Title 45, section 160.103: Definitions. eCFR https://www.ecfr.gov/current/title-45/ subtitle-A/subchapter-C/part-160/subpart-A section-160.103 (2021)

115. Code of Federal Regulations. Title 45, section 164.506: Uses and disclosures to carry out treatment, payment, or health care operations. eCFR https://www.ecfr.gov/ current/title-45/subtitle-A/subchapter-C/part-164/ subpart-E/section-164.506 (2021).

116. Code of Federal Regulations. Title 45, section 164.514: Other requirements relating to uses and disclosures of protected health information (b)(2). eCFR https: / www.ecfr.gov/current/title-45/subtitle-A/subchapter-C/ part-164/subpart-E/section-164.514\#p-164.514(b)(2) (2021).

117. Code of Federal Regulations. Title 45, section 164.514 Other requirements relating to uses and disclosures of protected health information (b)(1). eCFR https:// www.ecfr.gov/current/title-45/subtitle-A/subchapter-C/ part-164/subpart-E/section-164.514\#p-164.514(b)(1) (2021).

118. Code of Federal Regulations. Title 45, section 46.116 General requirements for informed consent (b)(9). eCFR https://www.ecfr.gov/current/title-45/subtitle-A/ subchapter-A/part-46/subpart-A/section-46.116\#p46.116(b)(9) (2021).

119. Code of Federal Regulations. Title 45 , section 46.116 General requirements for informed consent (d). eCFR https://www.ecfr.gov/current/title-45/subtitle-A/ subchapter-A/part-46/subpart-A/section-46.116\#p46.116(d) (2021).

120. Code of Federal Regulations. Title 45, section 46.116 General requirements for informed consent (c)(8). eCFR https://www.ecfr.gov/current/title-45/subtitle-A/ subchapter-A/part-46/subpart-A/section-46.116\#p46.116(c)(8) (2021).

121. Code of Federal Regulations. Title 45, section 46.116: General requirements for informed consent (c)(9). eCFR https://www.ecfr.gov/current/title-45/subtitle-A/ subchapter-A/part-46/subpart-A/section-46.116\#p46.116(c)(9) (2021).

122. Code of Federal Regulations. Title 45, section 46.110. Expedited review procedures for certain kinds of research involving no more than minimal risk, and for minor changes in approved research (b)(2). eCFR https://www.ecfr.gov/current/title-45/subtitle-A/ 
subchapter-A/part-46/subpart-A/section-46.110\#p46.110(b)(2) (2021)

123. Code of Federal Regulations. Title 45, section 46.116 General requirements for informed consent (f). eCFR https://www.ecfr.gov/current/title-45/subtitle-A/ subchapter-A/part-46/subpart-A/section-46.116\#p46.116(f) (2021)

124. Guerrini, C. J., McGuire, A. L. \& Majumder, M. A. Clearing complexity from the Common Rule NPRM J. Law Biosci. 3, 257-280 (2016).

125. California Supreme Court. Moore v. Regents of University of California. Court Listener https:// www. courtlistener.com/opinion/2608931/ moore-v-regents-of-university-of-california/ (1990)

126. US District Court, S.D. Florida, Miami Division. Greenberg v. Miami Children's Hospital Research Institute. Court Listener https://www. courtlistener.com/ opinion/2507167/greenberg-v-miami-childrenshospital-res-inst-inc/ (2003).

127. US District Court, E.D. Missouri, Eastern Division. Washington University v. Catalona. Court Listener https://www.courtlistener.com/opinion/2492481/ washington-university-v-catalona/ (2006)

128. 110th US Congress, The Genetic Information Nondiscrimination Act of 2008, Public Law 110-233. Congress.gov https://www.congress.gov/bill/110thcongress/house-bill/493/text (2008)

129. National Human Genome Research Institute. Genome Statute and Legislation Database. NHGRI https:// www.genome.gov/about-genomics/policy-issues/ Genome-Statute-Legislation-Database (2021).

130. California Legislative Information. California Consumer Privacy Act of 2018. California Legislative Information https://leginfo.legislature.ca.gov/faces/billTextClient. xhtml?bill_id=201720180SB 1121 (2018).

131. Office of the Attorney General. California Privacy Rights Act of 2020, Proposition 24, 1879 (19-0021A1). OAG https://oag.ca.gov/system/files/initiatives/pdfs/ $19-0021$ A $1 \% 20 \% 28$ Consumer $\% 2$ Privacy $\% 20-\% 20$ Version\%203\%29_1.pdf (2019).

132. Virginia's Legislative Information System. SB 1392 Consumer Data Protection Act. LIS https://lis.virginia. gov/cgi-bin/legp604.exe?211+sum+SB1392 (2021).

133. Colorado General Assembly. Colorado Privacy Act, SB 21-190, tit. 6, art. 1, part 13. Colorado General Assembly https://leg.colorado.gov/sites/default/files/ documents/2021A/bills/2021a 190 rer.pdf (2021).

134. Gavejian, J. C., Lazzarotti, J. J. \& Atrakchi, M. Virginia passes consumer privacy law; Other states may follow. National Law Review https://www. natlawreview.com/ article/virginia-passes-consumer-privacy-law-otherstates-may-follow (2021)

135. Brown, G. A. \& Lamoureux, C. Florida is the latest state to consider comprehensive data privacy legislation. National Law Review https://www.natlawreview.com/ article/florida-latest-state-to-consider-comprehensivedata-privacy-legislation (2021).

136. New York State Senate. New York Privacy Act: Senate Bill S6701. NY Senate https://www.nysenate.gov/ legislation/bills/2021/S6701 (2021)

137. Uniform Law Commission. Uniform Personal Data Protection Act. ULC https://www.uniformlaws.org/ HigherLogic/System/DownloadDocumentFile.ashx? DocumentFileKey=009e3927-eafa-3851-1c02 3a05f5891947 (2021).

138. Rippy, S. US state privacy legislation tracker. IAPP https://iapp.org/resources/article/us-state-privacylegislation-tracker (2021).

139. Yin, Z., Song, L., Clayton, E. W. \& Malin, B. A. Health and kinship matter: learning about direct-to-consume genetic testing user experiences via online discussions. PLOS ONE 15, e0238644 (2020).

140. Garrison, N. A. et al. A systematic literature review of individuals' perspectives on broad consent and data sharing in the United States. Genet. Med. 18, 663-671 (2016)

141. Kantarcioglu, M., Jiang, W., Liu, Y. \& Malin, B. A cryptographic approach to securely share and query genomic sequences. IEEE Trans. Inf. Technol. Biomed. 12, 606-617 (2008) This is the first study to use homomorphic encryption for privacy-preserving genomic computation.

142. Lauter, K., López-Alt, A. \& Naehrig, M. in Progress in Cryptology-LATINCRYPT 2014 (eds Aranha, D. F. \& Menezes, A.) 3-27 (Springer, 2015).

143. Wang, S. et al. HEALER: homomorphic computation of ExAct Logistic rEgRession for secure rare disease variants analysis in GWAS. Bioinformatics 32 211-218 (2016)

144. Tang, H. et al. Protecting genomic data analytics in the cloud: state of the art and opportunities. BMC Med. Genomics 9, 63 (2016).
145. Xie, W. et al. SecureMA: protecting participant privacy in genetic association meta-analysis. Bioinformatics 30, 3334-3341 (2014).

146. Cho, H., Wu, D. J. \& Berger, B. Secure genome-wide association analysis using multiparty computation. Nat. Biotechnol. 36, 547-551 (2018).

147. Jagadeesh, K. A., Wu, D. J., Birgmeier, J. A., Boneh, D. $\&$ Bejerano, G. Deriving genomic diagnoses without revealing patient genomes. Science 357, 692-695 (2017).

148. Jha, S., Kruger, L. \& Shmatikov, V. Towards practical privacy for genomic computation. Proc. 2008 IEEE Symp. Secur. Priv. 2008, 216-230 (2008). This is the first study to use SMC for practical privacy-preserving genomic computation.

149. Wu, X. et al. A novel privacy-preserving federated genome-wide association study framework and its application in identifying potential risk variants in ankylosing spondylitis. Brief. Bioinform. 22, bbaa090 (2021).

150. Froelicher, D. et al. Truly privacy-preserving federated analytics for precision medicine with multiparty homomorphic encryption. Nat. Commun. 12, 5910 (2021).

151. Canim, M., Kantarcioglu, M. \& Malin, B. Secure management of biomedical data with cryptographic hardware. IEEE Trans. Inf. Technol. Biomed. 16, 166-175 (2012) This is the first study to use cryptographic hardware for privacy-preserving genomic computation.

152. Berger, B. \& Cho, H. Emerging technologies towards enhancing privacy in genomic data sharing. Genome Biol. 20, 128 (2019).

153. Chen, F. et al. PRINCESS: Privacy-protecting Rare disease International Network Collaboration via Encryption through Software guard extensions. Bioinformatics 33, 871-878 (2017).

154. Sadat, M. N. et al. SAFETY: Secure gwAs in Federated Environment through a hYbrid Solution. IEEE/ACM Trans. Comput. Biol. Bioinform. 16, 93-102 (2019).

155. Kockan, C. et al. Sketching algorithms for genomic data analysis and querying in a secure enclave. Nat. Methods 17, 295-301 (2020)

156. Roberts, J. L., Pereira, S. \& McGuire, A. L. Should you profit from your genome? Nat. Biotechnol. 35, 18-20 (2017).

157. Ozercan, H. I., Ileri, A. M., Ayday, E. \& Alkan, C. Realizing the potential of blockchain technologies in genomics. Genome Res. 28, 1255-1263 (2018). This work shows the use of blockchain technologies for privacy-preserving personal genomic data sharing.

158. Zhang, Y. et al. Enabling privacy-preserving sharing of genomic data for GWASs in decentralized networks. Proc. 12th Int. Conf. Web Search Data Min. 2019 204-212 (2019)

159. Kuo, T-T., Gabriel, R. A. \& Ohno-Machado, L. Fair compute loads enabled by blockchain: sharing models by alternating client and server roles. J. Am. Med. Inform. Assoc. 26, 392-403 (2019).

160. Dankar, F. K. Data privacy through participant empowerment. Nat. Comput. Sci. 1, 175-176 (2021).

161. Grishin, D. et al. Citizen-centered, auditable and privacy-preserving population genomics. Nat. Comput Sci. 1, 192-198 (2021)

162. Office for Human Research Protections, US Department of Health and Human Services. International Compilation of Human Research Standards. HHS https://www.hhs.gov/ohrp/international/compilationhuman-research-standards/index.html (2020).

163. World Medical Association. Declaration of Helsinki: medical research involving human subjects. WMA https://www.wma.net/what-we-do/medical-ethics/ declaration-of-helsinki/ (2013)

164. Council for International Organizations of Medical Sciences. International ethical guidelines for healthrelated research involving humans. CIOMS https:/ cioms.ch/publications/product/international-ethicalguidelines-for-health-related-research-involving-humans (2016).

165. Congressional Research Service. EU data protection rules and US implications. EveryCRSReport.com https://www.everycrsreport.com/reports/IF10896.html (2020).

166. Ross, L., Zhou, K. \& Liu, T. China to strengthen regulation of human genetic resources. WilmerHale https://www.wilmerhale.com/en/insights/client-alerts/ 20200730-china-to-strengthen-regulation-of-humangenetic-resources (2020).

167. Chen, Y. \& Song, L. China: concurring regulation of cross-border genomic data sharing for statist control and individual protection. Hum. Genet. 137, 605-615 (2018)

168. Spice Route Legal. Cross-border transfers of genomic data: the Indian framework. Spice Route Legal https:/ spiceroutelegal.com/cross-border-transfers-of-genomicdata-the-indian-framework/ (2020).

169. de Vries, J. et al. Regulation of genomic and biobanking research in Africa: a content analysis of ethics guidelines, policies and procedures from 22 African countries. BMC Med. Ethics 18, 8 (2017).

170. Needham, K. \& Baldwin, C. China's gene giant harvests data from millions of women. Reuters https:/ www.reuters.com/investigates/special-report/ health-china-bgi-dna/ (2021).

171. Molla, R. Why DNA tests are suddenly unpopular. Vox https://www.vox.com/recode/2020/2/13/ $21129177 /$ consumer-dna-tests-23andme-ancestrysales-decline (2020).

172. Phillips, A. M. Only a click away - DTC genetics for ancestry, health, love... and more: a view of the business and regulatory landscape. Appl. Transl. Genom. 8 , 16-22 (2016)

173. Hazel, J. W. \& Slobogin, C. Who knows what, and when: a survey of the privacy policies proffered by US direct-to-consumer genetic testing companies. Cornell J. Law Public Policy 28, 35 (2018).

174. Laestadius, L. I., Rich, J. R. \& Auer, P. L. All your data (effectively) belong to us: data practices among directto-consumer genetic testing firms. Genet. Med. 19, 513-520 (2017)

175. US Food and Drug Administration. Direct-to-consumer tests. FDA https://www.fda.gov/medical-devices/ in-vitro-diagnostics/direct-consumer-tests (2019)

176. US Federal Trade Commission. Complaint, In re GeneLink, Inc. \& Foru Int'l Corp. FTC https://www.ftc.gov/ system/files/documents/cases/140512genelinkcmpt.pdf (2014).

177. Fair, L. DNA test kits: consider the privacy implications FTC https://www.consumer.ftc.gov/blog/2017/12/ dna-test-kits-consider-privacy-implications (2017)

178. US Federal Trade Commission. Direct-to-consumer genetic tests. FTC https://www.consumer.ftc.gov/ articles/0166-direct-consumer-genetic-tests (2018).

179. Jillson, E. Selling genetic testing kits? Read on FTC https://www.ftc. gov/news-events/blogs/ business-blog/2019/03/selling-genetic-testing-kitsread (2019)

180. Cheon, J. H., Kim, M. \& Lauter, K. in Financial Cryptography and Data Security (eds Böhme, R. \& Okamoto, T.) 194-212 (Springer, 2015).

181. Baldi, P., Baronio, R., De Cristofaro, E., Gasti, P. $\&$ Tsudik, G. Countering gattaca: efficient and secure testing of fully-sequenced human genomes. Proc. 18th ACM Conf. Comput. Commun. Secur. 2011, 691-702 (2011).

182. He, D. et al. Identifying genetic relatives without compromising privacy. Genome Res. 24, 664-672 (2014).

183. Greytak, E. M., Moore, C. \& Armentrout, S. L. Genetic genealogy for cold case and active investigations. Forensic Sci. Int. 299, 103-113 (2019).

184. National College of State Legislatures. DNA arrestee laws (NCSL, 2013)

185. Federal Bureau of Investigation. The FBl's Combined DNA Index System (CODIS) hits major milestone. FBI https://www.fbi.gov/news/pressrel/press-releases/ the-fbis-combined-dna-index-system-codis-hits-majormilestone (2021)

186. Federal Bureau of Investigation. CODIS-NDIS statistics. FBI https://www.fbi.gov/services/laboratory/ biometric-analysis/codis/ndis-statistics (2021).

187. Hill, K. \& Murphy, H. Your DNA profile is private? A Florida judge just said otherwise. New York Times https://www.nytimes.com/2019/11/05/business/ dna-database-search-warrant.html (2019).

188. Code of Federal Regulations. Title 42, section 241. Research and investigations generally (d)(1)(E). eCFR https://www.govinfo.gov/content/pkg/USCODE-2020title42/html/USCODE-2020-title42-chap6A-subchapllpartA-sec241.htm (2020).

189. Wolf, L. E. \& Beskow, L. M. Certificates of confidentiality: mind the gap. Utah Law Rev. 2021, 937-950 (2021).

190. Haag, M. FamilyTreeDNA admits to sharing genetic data with the FBI. New York Times https://www.nytimes.com/ 2019/02/04/business/family-tree-dna-fbi.html (2019).

191. Aldhous, P. A court tried to force Ancestry.com to open up its DNA database to police. The company said no. BuzzFeed News https://www.buzzfeednews.com/article peteraldhous/ancestry-dna-database-search-warrant (2020). 
192. US Department of Justice. Interim policy forensic genetic genealogical DNA analysis and searching. Department of Justice https://www.justice.gov/olp page/file/1204386/download (2019).

193. Sacramento County District Attorney's Office. Memorandum of understanding: investigative genetic genealogy searching. CHIA https://chia187. wildapricot. org/page-1841969 (2019).

194. Granja, R. Long-range familial searches in recreational DNA databases: expansion of affected populations, the participatory turn, and the co-production of biovalue. N Genet Soc. 40, 331-352 (2021).

195. Scudder, N., Daniel, R., Raymond, J. \& Sears, A. Operationalising forensic genetic genealogy in an Australian context. Forensic Sci. Int. 316, 110543 (2020).

196. Kale, G. Ayday, E. \& Tastan, O. A utility maximizing and privacy preserving approach for protecting kinship in genomic databases. Bioinformatics 34, 181-189 (2018).

This study optimizes SNP masking while mitigating kinship privacy risks stemming from familial searches.

197. Bednar, K., Spiekermann, S. \& Langheinrich, M. Engineering privacy by design: are engineers ready to live up to the challenge? Inf. Soc. 35, 122-142 (2019).

198. Oprisanu, B., Dessimoz, C. \& De Cristofaro, E. How much does GenoGuard really "guard"? An empirical analysis of long-term security for genomic data. Proc. 18th ACM Workshop Priv. Electron. Soc. 2019. 93-105 (2019).

\section{Acknowledgements}

The authors would like to thank their colleagues at the Center for Genetic Privacy and Identity in Community Settings (GetPreCiSe) at Vanderbilt University Medical Center for their constructive feedback. This work was mainly sponsored by GetPreCiSe, a Center for Excellence in Ethical, Legal and Social Implications (ELSI) Research, through a grant from the National Human Genome Research Institute, Nationa Institutes of Health (RM1HG009034). This work was also funded, in part, by the following grants from the Nationa Institutes of Health: R01HG006844 and R01LM009989.

\section{Author contributions}

Z.W. and J.W.H. conducted the literature review and drafted the technical and legal parts, respectively. E.W.C. and B.A.M. provided the motivation for this work and designed the organization and structure of the article. E.W.C., Y.V., M.K. and B.A.M provided detailed edits and critical suggestions on the organization and structure of the article. All authors wrote the manuscript. Z.W. and J.W.H. contributed equally to all aspects of the article. All authors read and approved the final manuscript.

\section{Competing interests}

The authors declare no competing interests.

Publisher's note

Springer Nature remains neutral with regard to jurisdictional claims in published maps and institutional affiliations.

\section{RELATED LINKS}

1000 Genomes Project: https://www.internationalgenome.org/ All of Us Research Program: https://allofus.nih.gov/ Ancestry.com: https://www.ancestry.com Beacon Network: https://beacon-network.org/ dbGaP: http://www.ncbi.nlm.nih.gov/gap eMERGE network: https://emerge-network.org/ FamilyTreeDNA: https://www.familytreedna.com GEDmatch: http://gedmatch.com

(C) Springer Nature Limited 2022, corrected publication 2022 